\title{
The transient analysis of a conducting crack in magneto- electro-elastic half-space under anti-plane mechanical and in-plane electric and magnetic impacts
}

Received: 17 October 2013 / Accepted: 10 July 2014 / Published online: 23 August 2014

(C) The Author(s) 2014. This article is published with open access at Springerlink.com

\begin{abstract}
This paper investigated the fracture behavior of a magneto-electro-elastic material subjected to transient electrical, magnetic and mechanical loads. The "smart" medium contains a straight-line crack, which is parallel to its poling direction and free boundary surface. The Fourier and Laplace transform techniques are used to reduce the problem to the solution of one Fredholm integral equation in Laplace domain and second equation in real domain. The Laplace inversion yields the result in the time domain. The equation in real domain is solved exactly. The semipermeable crack-face magneto-electric boundary conditions are utilized. Field intensity factors of stress, electric displacement, magnetic induction, crack displacement, electric and magnetic potentials and the energy release rate are determined. The electric displacement and magnetic induction of crack interior are discussed. Strong coupling between stress and electric and magnetic field near crack tips has been found. Numerical results are presented, and some conclusions are drawn.
\end{abstract}

Keywords MEEMs half-space · Dielectric crack · Semipermeable condition · Fourier and Laplace transforms · Transient problem $\cdot$ Field intensity factor $\cdot$ Energy release rate $\cdot$ Exact solution

\section{Introduction}

Many piezo-electro-magnetic devices may experience transient loads. For example, devices such as phase change transducers and pulse generators for igniter and high voltage transformers are almost routinely subjected to very large voltages over very short intervals of time. The magneto-electro-mechanical impact loading generates elastic waves through the structure, which are reflected and refracted at cracks, causing the local stresses to increase beyond its corresponding value under static loads of the same magnitude. This intensification of local stress could initiate unstable motion of the crack and result in fracture of the structure. Therefore, many authors have studied the dynamic fracture of piezo-electric materials. Ueda [1], Nishioka et al. [2], Jin et al. [3], Li and Tang [4], He [5], Ricci et al. [6], Chen and Worswick [7], Wang and Mai [8] and Li [9] have analyzed fracture problems, which are limited to insulating crack. Several earlier works on magnetoelectro-elastic and piezo-electric solids with cracks: Tian and Rajapakse $[10,11]$ are worth mentioning. In the first paper, the solutions for plane static problem of impermeable cracks have been formulated in terms of set of singular integral equations using Stroh formalism [12] and which are solved by a numerical integration technique. In the second paper, an integral equation formulation has been developed to analyze of a static response of impermeable penny-shaped cracks located at the interface of a piezo-electric bi-material system, and numerical scheme has been used to obtain the results. Clearly, there is a need to investigate the dielectric and magnetic conducting cracks in magneto-electro-elastic material (MEEMs) under transient electromechanical impact. Motivated by this consideration, this paper investigates a MEEMs half-space with an electrically 
and magnetically conducting crack under an anti-plane mechanical and an in-plane electro-magnetic impact. Exact solutions in analytical form are obtained. Since all the formulas in this paper are obtained in explicit expressions, this study may serve as a benchmark for further investigations in MEEMs.

\section{Basic equations}

For a linear MEEMs under anti-plane shear coupled with in-plane electric and magnetic fields, there is only the non-trivial anti-plane displacement $w$, that is,

$$
u_{x}=0, u_{y}=0, u_{z}=w(x, y, t)
$$

strain components $\gamma_{x z}$ and $\gamma_{y z}$, that is,

$$
\gamma_{x z}=\frac{\partial w}{\partial x}, \gamma_{y z}=\frac{\partial w}{\partial y}
$$

stress components $\tau_{x z}$ and $\tau_{y z}$, in-plane electric and magnetic potentials $\phi$ and $\psi$, which define electric and magnetic field components $E_{x}, E_{y}, H_{x}$ and $H_{y}$ as follows

$$
E_{x}=-\frac{\partial \phi}{\partial x}, \quad E_{y}=-\frac{\partial \phi}{\partial y}, \quad H_{x}=-\frac{\partial \psi}{\partial x}, \quad H_{y}=-\frac{\partial \psi}{\partial y}
$$

and electrical displacement components $D_{x}, D_{y}$, and magnetic induction components $B_{x}, B_{y}$ with all field quantities being the functions of coordinates $x$ and $y$ and time $t$.

The generalized strain-displacement relations (2) and (3) have the form:

$$
\gamma_{\alpha z}=w_{, \alpha}, \quad E_{\alpha}=-\phi_{, \alpha}, \quad H_{\alpha}=-\psi_{, \alpha}
$$

where $\alpha=x, y$ and $w_{, \alpha}=\partial w / \partial \alpha$.

For linear MEEMs, the coupled constitutive relations can be written in the matrix form

$$
\left[\tau_{\alpha z}, D_{\alpha}, B_{\alpha}\right]^{\mathrm{T}}=\mathbf{C}\left[\gamma_{\alpha z},-E_{\alpha},-H_{\alpha}\right]^{\mathrm{T}}
$$

where the superscript $\mathrm{T}$ denotes the transpose of a matrix and

$$
\mathbf{C}=\left[\begin{array}{lll}
c_{44} & e_{15} & q_{15} \\
e_{15} & -\varepsilon_{11} & -d_{11} \\
q_{15} & -d_{11} & -\mu_{11}
\end{array}\right]
$$

where $c_{44}$ is the shear modulus along the $z$-direction, which is the direction of poling and principal axis of elastic symmetry, perpendicular to the isotropic plane $(x, y), \varepsilon_{11}$ and $\mu_{11}$ are dielectric permittivity and magnetic permeability coefficients, respectively, $e_{15}, q_{15}$ and $d_{11}$ are piezo-electric, piezo-magnetic and magneto-electric coefficients, respectively.

The mechanical dynamic equilibrium equation, the charge and current conservation equations, in the absence of the body force electric and magnetic charge densities, can be written as

$$
\tau_{z \alpha, \alpha}=\rho \ddot{w}, \quad D_{\alpha, \alpha}=0, \quad B_{\alpha, \alpha}=0, \quad \alpha=x, y
$$

where $\rho$ is the mass density of material and double dot denotes second-order derivative with respect to time. Equations (1)-(7) define the coupled fields in a transversely isotropic MEEMs (Parton and Kudryavtsev [13]). In view of Eqs. (4) and (5), Eq. (7) can be reduced to

$$
\mathbf{C}\left[\nabla^{2} w, \nabla^{2} \phi, \nabla^{2} \psi\right]^{\mathrm{T}}=[\rho \ddot{w}, 0,0]^{\mathrm{T}}
$$

where $\nabla^{2}=\partial^{2} / \partial x^{2}+\partial^{2} / \partial y^{2}$ is the two-dimensional Laplace's operator. Since $|\mathbf{C}| \neq 0$, one can decouple the equations (8)

$$
\nabla^{2} w=c_{3}^{-2} \ddot{w}, \quad \nabla^{2} \phi=\alpha \nabla^{2} w, \quad \nabla^{2} \psi=\beta \nabla^{2} w
$$


where

$$
\begin{aligned}
& c_{3}=\sqrt{\frac{\tilde{c}_{44}}{\rho}}, \quad \tilde{c}_{44}=c_{44}+\alpha e_{15}+\beta q_{15} \\
& \alpha=\frac{\mu_{11} e_{15}-d_{11} q_{15}}{\varepsilon_{11} \mu_{11}-d_{11}^{2}}, \quad \beta=\frac{\varepsilon_{11} q_{15}-d_{11} e_{15}}{\varepsilon_{11} \mu_{11}-d_{11}^{2}}
\end{aligned}
$$

Note that $c_{3}$ is the shear wave velocity in magneto-electro-elastic body and $\tilde{c}_{44}$ is the piezo-electromagnetically stiffened elastic constant.

Introducing two new functions $\phi_{1}$ and $\psi_{1}$ such that:

$$
\phi_{1}=\phi-\alpha w, \quad \psi_{1}=\psi-\beta w
$$

we obtain

$$
\nabla^{2} \phi_{1}=0, \quad \nabla^{2} \psi_{1}=0
$$

with $\phi_{1}$ and $\psi_{1}$ being harmonic functions.

\section{Formulation of the crack problem}

Consider MEEMs half-space containing a Griffith crack of length $2 a$, parallel to the surface of a half-space, which is subjected to electrical, magnetic and mechanical loads. The crack is located along the $x$-axis from $-a$ to $a$ at a depth $h$ from the loaded surface with a rectangular coordinate system, as shown in Fig. 1. The MEEMs half-space is poled in the direction of the $z$-axis, which guarantees the piezo-electro-magneto-elastic material transversely isotropic properties.

In pure elastic solid, the exact solution of the above problem may be straightforwardly obtained (Ma and Chen [14]). The piezo-electro-magneto-elastic case is more complicated, with most of the difficulty stemming from the imposition of the electrical and magnetic boundary conditions.

To solve the crack problem in linear elastic solid, the superposition technique is usually used. Thus, we first solve the magneto-electro-elastic field problem without cracks in the medium under electrical, magnetic and mechanical loads. This elementary solution is:

$$
\begin{aligned}
& \tau_{y z}=\tau_{0} \\
& D_{y}=\bar{D}=\left\{\begin{array}{l}
D_{0}, \quad \text { Case I } \\
\frac{e_{15}}{c_{44}} \tau_{0}+\left(\varepsilon_{11}+\frac{\left(e_{15}\right)^{2}}{c_{44}}\right) E_{0}+\left(d_{11}+\frac{e_{15} q_{15}}{c_{44}}\right) H_{0}, \quad \text { Case II }
\end{array}\right. \\
& B_{y}=\bar{B}=\left\{\begin{array}{l}
B_{0}, \quad \text { Case I } \\
\frac{q_{15}}{c_{44}} \tau_{0}+\left(d_{11}+\frac{e_{15} q_{15}}{c_{44}}\right) E_{0}+\left(\mu_{11}+\frac{\left(q_{15}\right)^{2}}{c_{44}}\right) H_{0}, \quad \text { Case II }
\end{array}\right.
\end{aligned}
$$

Then, we use equal and opposite quantities as the crack surface traction and utilize the unknowns $d_{0}$ and $b_{0}$. Thus, in this study, $-\tau_{0},-\left(\bar{D}-d_{0}\right)$ and $-\left(\bar{B}-b_{0}\right)$ are, respectively, mechanical, electrical and magnetic loadings applied on the cracks surfaces (the so-called perturbation problem). The boundary conditions can be written as follows:
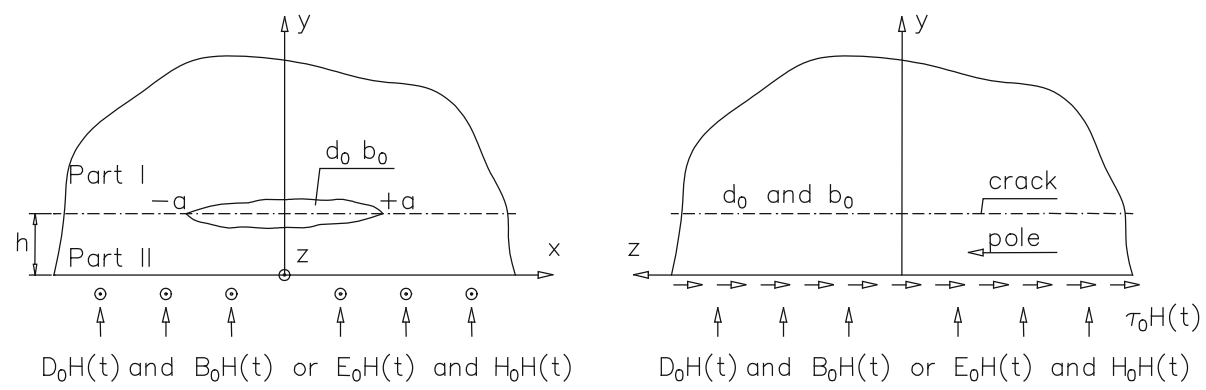

Fig. 1 The MEEMs half-space with a crack parallel to its surface under an anti-plane mechanical and in-plane electrical and magnetic impact. Inside the crack the unknown electro-magnetic field appears ( $d_{0}$ and $b_{0}$ are unknown to be determined) 


$$
\begin{aligned}
& \tau_{z y}(x, h \pm, t)=-\tau_{0} H(t) \\
& \left.D_{y}(x, h \pm, t)=-D H(t)\right\}, \quad|x|<a \\
& B_{y}(x, h \pm, t)=-B H(t) \\
& \tau_{z y}(x, h+, t)=\tau_{z y}(x, h-, t) \\
& \left.D_{y}(x, h+, t)=D_{y}(x, h-, t)\right\}, \quad|x|<\infty \\
& B_{y}(x, h+, t)=B_{y}(x, h-, t) \\
& w(x, h+, t)=w(x, h-, t) \\
& \phi(x, h+, t)=\phi(x, h-, t)\}, \quad|x|>a \\
& \psi(x, h+, t)=\psi(x, h-, t) \\
& \tau_{z y}(x, 0, t)=0 \\
& \left.D_{y}(x, 0, t)=0\right\}, \quad|x|<\infty \\
& B_{y}(x, 0, t)=0
\end{aligned}
$$

where $H(t)$ is the Heaviside unit step function defined as:

$$
H(t)= \begin{cases}0 ; & t \leq 0 \\ 1 ; & t>0\end{cases}
$$

Of course, in perturbation problem, the surface of the half-space is free. The electric displacement $d_{0}$ and the magnetic induction $b_{0}$ inside the crack are unknowns and should be further determined. This field is uniform if the applied loading $\left(-\tau_{0}, \bar{D}, \bar{B}\right)$ is uniform (Rogowski [15]), but its changes with time in transient problem. Thus, on the crack surfaces, the fields

$$
\begin{gathered}
D=\bar{D}-d_{0}(t) \\
B=\bar{B}-b_{0}(t)
\end{gathered}
$$

are used in Eq. (13a). In the formulation of boundary conditions, we considered a flaw in the medium as a cleavage crack of zero gap width (the so-called mathematical crack). In fracture mechanics experiments, however, the flaw in a specimen is usually cut with a tool of finite thickness. Thus, flaws in the experiments are not like cleavage cracks of zero gap width, but rather like notches with a finite width. Suppose the thickness $\delta$ of the notch is sufficiently small, except near the tip of the notch, the gradient of the notch opening along the notch is small. Along the $y$ direction, the electric field $E_{y}^{c}$ and the electric displacement $d_{0}$, the magnetic field $H_{y}^{c}$ and magnetic induction $b_{0}$ on the upper notch surface can be written as (Rogowski [15]):

$$
\begin{gathered}
E_{y}^{c}=-\frac{\phi^{+}-\phi^{-}}{2 \delta(x)}, \quad d_{0}=\varepsilon_{c} E_{y}^{c} \\
H_{y}^{c}=-\frac{\psi^{+}-\psi^{-}}{2 \delta(x)}, \quad b_{0}=\mu_{c} H_{y}^{c}
\end{gathered}
$$

where $\phi^{ \pm} \equiv \phi(x, h \pm, t)$ and $\delta(x)$ describe the shape of the notch and $\varepsilon_{c}, \mu_{c}$ are the dielectric permittivity and magnetic permeability of crack interior. Equation (18) is valid if we suppose the notch profile $\delta(x)$ is sufficiently small, except the notch tips, the gradient of notch opening along the notch is small. Since the notch profile $\delta(x)$ is sufficiently small, the crack solution can still approximately applied to the notch problem. If we assume the elliptic notch profile such that:

$$
\delta(x)=\frac{\delta_{0}}{a} \sqrt{a^{2}-x^{2}}
$$

where $\delta_{0}$ is the half-thickness of the notch at $x=0$, we obtain

$$
\begin{aligned}
& \frac{2 d_{0} \delta_{0}}{a \varepsilon_{c}} \sqrt{a^{2}-x^{2}}=-\left(\phi^{+}-\phi^{-}\right) \\
& \frac{2 b_{0} \delta_{0}}{a \mu_{c}} \sqrt{a^{2}-x^{2}}=-\left(\psi^{+}-\psi^{-}\right)
\end{aligned}
$$

Equation (20) form two coupling linear equations with respect to $d_{0}$ and $b_{0}$ since $\phi^{+}$and $\phi^{-}$depend linearly on these quantities. If the boundary conditions consist electric displacement $D$ and magnetic induction $B$, as in Eq. (13a), it is convenient to introduce two new unknown functions instead of $\phi_{1}$ and $\psi_{1}$, such that: 


$$
\begin{aligned}
& \chi=-\varepsilon_{11} \phi_{1}-d_{11} \psi_{1} \\
& \eta=-d_{11} \phi_{1}-\mu_{11} \psi_{1}
\end{aligned}
$$

Then

$$
\begin{aligned}
& \phi_{1}=e_{1} \chi+e_{2} \eta \\
& \psi_{1}=e_{2} \chi+e_{3} \eta
\end{aligned}
$$

where

$$
e_{1}=-\frac{\mu_{11}}{\varepsilon_{11} \mu_{11}-\left(d_{11}\right)^{2}}, \quad e_{2}=\frac{d_{11}}{\varepsilon_{11} \mu_{11}-\left(d_{11}\right)^{2}}, \quad e_{3}=-\frac{\varepsilon_{11}}{\varepsilon_{11} \mu_{11}-\left(d_{11}\right)^{2}}
$$

The stress, electric potential $\phi$ and displacement $D_{k}$, magnetic potential $\psi$ and induction $B_{k}$ and governing integral equations are:

$$
\begin{aligned}
\tau_{z k} & =\tilde{c}_{44} w_{, k}-\alpha D_{k}-\beta B_{k} \\
\phi & =e_{1} \chi+e_{2} \eta+\alpha w \\
\psi & =e_{2} \chi+e_{3} \eta+\beta w \\
D_{k} & =\chi_{, k} \\
B_{k} & =\eta_{, k}, \quad k=x, y \\
\nabla^{2} w & =\rho \ddot{w} c_{3}^{-2} \\
\nabla^{2} \chi & =0, \quad \nabla^{2} \eta=0
\end{aligned}
$$
0 .

The two last equations (30) are equivalent to Eqs. (12) and (22) since $e_{1} e_{3}-\left(e_{2}\right)^{2}=1 /\left(\varepsilon_{11} \mu_{11}-\left(d_{11}\right)^{2}\right) \neq$

Note that the inverse of matrix $C$ is defined by parameters $\alpha, \beta, \tilde{c}_{44}$ and $e_{1}, e_{2}, e_{3}$ as follows

$$
C^{-1}=\frac{1}{\tilde{c}_{44}}\left[\begin{array}{lll}
1 & \alpha & \beta \\
\alpha & \alpha^{2}+\tilde{c}_{44} e_{1} & \alpha \beta+\tilde{c}_{44} e_{2} \\
\beta & \alpha \beta+\tilde{c}_{44} e_{2} & \beta^{2}+\tilde{c}_{44} e_{3}
\end{array}\right]
$$

and is the matrix generalized compliances of MEEMs. These material parameters will be appeared in our solutions.

\section{Fredholm integral equations of the second kind}

Define the Laplace and Fourier transforms pair by the equations:

$$
\begin{aligned}
f^{*}(p) & =\int_{0}^{\infty} f(t) e^{-p t} \mathrm{~d} t, \quad f(t)=\frac{1}{2 \pi i} \int_{B r} f^{*}(p) e^{p t} \mathrm{~d} p \\
\hat{f}(s) & =\int_{0}^{\infty} f(x) \cos (s x) \mathrm{d} x, \quad f(x)=\frac{2}{\pi} \int_{0}^{\infty} \hat{f}(s) \cos (s x) \mathrm{d} s
\end{aligned}
$$

in which $\mathrm{Br}$ denotes the Bromwich path of integration. The time- and $x$-dependences in Eqs. (24)-(30) are eliminated by the application of Eqs. (32a) and (32b).

Considering the symmetry about $y$-axis in the functions $w, \phi$ and $\psi$ (or $w, \chi$ and $\eta$ ), the Fourier cosine transform is then applied, resulting in:

$$
\begin{aligned}
& \left.\begin{array}{l}
\hat{w}^{*}(s, y, p)=A_{1}(s, p) e^{-\gamma y} \\
\hat{\chi}^{*}(s, y, p)=B_{1}(s, p) e^{-s y} \\
\hat{\psi}^{*}(s, y, p)=C_{1}(s, p) e^{-s y}
\end{array}\right\}, \quad y>h \\
& \hat{w}^{*}(s, y, p)=A_{2}(s, p) e^{-\gamma y}+A_{3}(s, p) e^{\gamma y} \\
& \left.\begin{array}{l}
\hat{\chi}^{*}(s, y, p)=B_{2}(s, p) e^{-s y}+B_{3}(s, p) e^{s y} \\
\hat{\psi}^{*}(s, y, p)=C_{2}(s, p) e^{-s y}+C_{3}(s, p) e^{s y}
\end{array}\right\}, \quad 0 \leq y<h
\end{aligned}
$$


in which

$$
\gamma(s, p)=\sqrt{s^{2}+p^{2} c_{3}^{-2}}
$$

In the domain $y>h$, the solution has the form (33) to ensure the regularity condition at infinity. The transforms of Eqs. (24)-(28) yield:

$$
\begin{aligned}
& \hat{\phi}^{*}(s, y, p)=e_{1} \hat{\chi}^{*}(s, y, p)+e_{2} \hat{\eta}^{*}(s, y, p)+\alpha \hat{w}^{*}(s, y, p) \\
& \hat{\psi}^{*}(s, y, p)=e_{2} \hat{\chi}^{*}(s, y, p)+e_{3} \hat{\eta}^{*}(s, y, p)+\beta \hat{w}^{*}(s, y, p) \\
& \hat{\tau}_{z y}^{*}=\tilde{c}_{44} \hat{w}_{, y}^{*}-\alpha \hat{D}_{y}^{*}-\beta \hat{B}_{y}^{*} \\
& \hat{D}_{y}^{*}=\hat{\chi}_{, y}^{*}, \quad \hat{B}_{y}^{*}=\hat{\eta}_{, y}^{*}
\end{aligned}
$$

The unknown functions $A_{i}(s, p), B_{i}(s, p)$ and $C_{i}(s, p), i=1,2,3$ are obtained from the boundary conditions (13b) and (15), which in transform domains may be written as:

$$
\begin{aligned}
& \hat{\tau}_{z y}^{*}(s, h+, p)=\hat{\tau}_{z y}^{*}(s, h-, p) \\
& \hat{D}_{y}^{*}(s, h+, p)=\hat{D}_{y}^{*}(s, h-, p) \\
& \hat{B}_{y}^{*}(s, h+, p)=\hat{B}_{y}^{*}(s, h-, p) \\
& \hat{\tau}_{z y}^{*}(s, 0, p)=0 \\
& \hat{D}_{y}^{*}(s, 0, p)=0 \\
& \hat{B}_{y}^{*}(s, 0, p)=0
\end{aligned}
$$

The result is:

$$
\begin{aligned}
& A_{1}(s, p)=\hat{f}(s, p)\left(e^{-\gamma h}-e^{\gamma h}\right) \\
& B_{1}(s, p)=\hat{g}(s, p)\left(e^{-s h}-e^{s h}\right) \\
& C_{1}(s, p)=\hat{h}(s, p)\left(e^{-s h}-e^{s h}\right) \\
& A_{2}(s, p)=A_{3}(s, p)=\hat{f}(s, p) e^{-\gamma h} \\
& B_{2}(s, p)=B_{3}(s, p)=\hat{g}(s, p) e^{-s h} \\
& C_{2}(s, p)=C_{3}(s, p)=\hat{h}(s, p) e^{-s h}
\end{aligned}
$$

where $\hat{f}(s, p), \hat{g}(s, p)$ and $\hat{h}(s, p)$ are new unknown functions, Finally, the solutions for the half-space $y \geq 0$ in Laplace domain are:

$$
\begin{aligned}
& w^{*}(x, y, p)=-\frac{2}{\pi} \int_{0}^{\infty} \hat{f}(s, p)\left[\operatorname{sgn}(y-h) e^{-\gamma|y-h|}-e^{-\gamma(y+h)}\right] \cos (s x) \mathrm{d} s \\
& \chi^{*}(x, y, p)=-\frac{2}{\pi} \int_{0}^{\infty} \hat{g}(s, p)\left[\operatorname{sgn}(y-h) e^{-s|y-h|}-e^{-s(y+h)}\right] \cos (s x) \mathrm{d} s \\
& \eta^{*}(x, y, p)=-\frac{2}{\pi} \int_{0}^{\infty} \hat{h}(s, p)\left[\operatorname{sgn}(y-h) e^{-s|y-h|}-e^{-s(y+h)}\right] \cos (s x) \mathrm{d} s \\
& \tau_{z y}^{*}(x, y, p)=\frac{2}{\pi} \tilde{c}_{44} \int_{0}^{\infty} \gamma \hat{f}(s, p)\left(e^{-\gamma|y-h|}-e^{-\gamma(y+h)}\right) \cos (s x) \mathrm{d} s+ \\
& \quad-\frac{2}{\pi} \int_{0}^{\infty} s[\alpha \hat{g}(s, p)+\beta \hat{h}(s, p)]\left(e^{-s|y-h|}-e^{-s(y+h)}\right) \cos (s x) \mathrm{d} s
\end{aligned}
$$




$$
\begin{aligned}
& D_{y}^{*}(x, y, p)=\frac{2}{\pi} \int_{0}^{\infty} s \hat{g}(s, p)\left(e^{-s|y-h|}-e^{-s(y+h)}\right) \cos (s x) \mathrm{d} s \\
& B_{y}^{*}(x, y, p)=\frac{2}{\pi} \int_{0}^{\infty} s \hat{h}(s, p)\left(e^{-s|y-h|}-e^{-s(y+h)}\right) \cos (s x) \mathrm{d} s
\end{aligned}
$$

where $\operatorname{sgn}(y-h)=+1$ or $\operatorname{sgn}(y-h)=-1$ for $y>h$ or $y<h$, respectively.

The potentials $\phi^{*}$ and $\psi^{*}$ are obtained from Eq. (36). The unknown functions $\hat{f}(s, p), \hat{g}(s, p)$ and $\hat{h}(s, p)$ can be obtained from the mixed boundary conditions (13a) and (14) which yield:

$$
\begin{aligned}
& \left.\begin{array}{l}
\frac{2}{\pi} \int_{0}^{\infty} \gamma \hat{f}(s, p)\left(1-e^{-2 \gamma h}\right) \cos (s x) \mathrm{d} s=-\frac{\tau_{0}+D \alpha+B \beta}{p \tilde{c}_{44}} \\
\frac{2}{\pi} \int_{0}^{\infty} s \hat{g}(s, p)\left(1-e^{-2 s h}\right) \cos (s x) \mathrm{d} s=-\frac{D}{p} \\
\frac{2}{\pi} \int_{0}^{\infty} s \hat{h}(s, p)\left(1-e^{-2 s h}\right) \cos (s x) \mathrm{d} s=-\frac{B}{p}
\end{array}\right\}, \quad|x|<a \\
& \int_{0}^{\infty} \hat{f}(s, p) \cos (s x) \mathrm{d} s=0 \\
& \left.\int_{0}^{\infty} \hat{g}(s, p) \cos (s x) \mathrm{d} s=0\right\}, \quad|x|>a \\
& \int_{0}^{\infty} \hat{h}(s, p) \cos (s x) \mathrm{d} s=0
\end{aligned}
$$

Here $D=\bar{D}-d_{0}^{*}(p)$ and $B=\bar{B}-b_{0}^{*}(p)$ (the asterisk $*$ in $D$ and $B$ is omitted to the sake of brevity). The integral equations (41) may be rewritten as:

$$
\frac{2}{\pi} \int_{0}^{\infty}\left\{\begin{array}{l}
\frac{\gamma}{s} \hat{f}(s, p) \\
\hat{g}(s, p) \\
\hat{h}(s, p)
\end{array}\right\}\left\{\begin{array}{l}
1-e^{-2 \gamma h} \\
1-e^{-2 s h} \\
1-e^{-2 s h}
\end{array}\right\} \sin (s x) \mathrm{d} s=-\left\{\begin{array}{l}
\frac{\tau_{0}+D \alpha+B \beta}{D \tilde{c}_{44}} x \\
\frac{D}{p} x \\
\frac{B}{p} x
\end{array}\right\}
$$

Note that both $\hat{g}(s, p)$ and $\hat{h}(s, p)$ have the same nature being proportional to $D=\bar{D}-d_{0}(p)$ and $B=\bar{B}-b_{0}(p)$, respectively. We introduce the integral representation of the unknown functions:

$$
\frac{2}{\pi}\left\{\begin{array}{l}
\hat{f}(s, p) \\
\hat{g}(s, p) \\
\hat{h}(s, p)
\end{array}\right\}=-\left\{\begin{array}{l}
\frac{\tau_{0}+D \alpha+B \beta}{D} p \tilde{c} 44 \\
\frac{D}{p} \\
\frac{B}{p}
\end{array}\right\} \int_{0}^{\infty}\left\{\begin{array}{l}
f(u, p) \\
g(u, p) \\
h(u, p)
\end{array}\right\} u J_{0}(s u) \mathrm{d} u
$$

where $J_{0}(s u)$ is the Bessel function of the first kind and zero order and $f(u, p), g(u, p)$ and $h(u, p)$ are new auxiliary functions. This representation satisfies equations (42) automatically and converts equations (41) to the Abel integral equations, which can be inverted explicitly. Here we omit details for saving space. As a result of well-known mathematical techniques, the Fredholm integral equations of the second kind are obtained as follows: 


$$
\begin{aligned}
& f(u, p)+\int_{0}^{a} f(v, p) K(u, v, p) \mathrm{d} v=1 \\
& g(u)-\int_{0}^{a} g(v) K(u, v) \mathrm{d} v=1 \\
& h(u)-\int_{0}^{a} h(v) K(u, v) \mathrm{d} v=1
\end{aligned}
$$

with the kernels

$$
\begin{aligned}
& K(u, v, p)=K_{d}(u, v, p)-K(u, v) \\
& K_{d}(u, v, p)=v \int_{0}^{\infty}(\gamma(s, p)-s)\left(1-e^{-2 \gamma h}\right) J_{0}(s u) J_{0}(s v) \mathrm{d} s \\
& K(u, v)=v \int_{0}^{\infty} s e^{-2 s h} J_{0}(s u) J_{0}(s v) \mathrm{d} s
\end{aligned}
$$

Note that $g(u, p)=g(u), h(u, p)=h(u)$ since the kernel function $K(u, v)$ is independent on $p$. Additionally, $g(u)=h(u)$. But $\hat{g}(s, p)$ and $\hat{h}(s, p)$ in Eq. (44) are dissimilar since are proportional to $\bar{D}-d_{0}(p)$ and $\bar{B}-b_{0}(p)$, respectively, and $d_{0}(p)$ and $b_{0}(p)$ are dissimilar functions. Note that $K(u, v, p)$ is expressed as the difference of transient part $K_{d}(u, v, p)$ and static part $K(u, v)$. [16]):

The kernel functions $K(u, v)$ may be presented in more useful form. Using the Neuman's theorem (Watson

$$
J_{0}(s u) J_{0}(s v)=\frac{1}{\pi} \int_{0}^{\pi} J_{0}(s R) d \alpha, \quad R^{2}=u^{2}+v^{2}-2 u v \cos \alpha
$$

and the integral (Gradshteyn and Ryzhik [17])

$$
\int_{0}^{\infty} s J_{0}(s R) e^{-2 s h} \mathrm{~d} s=\frac{2 h}{\left[R^{2}+(2 h)^{2}\right]^{3 / 2}}
$$

the kernel function becomes

$$
\begin{aligned}
& K(u, v)=\frac{4 h v}{\pi l^{3 / 2}} \int_{0}^{\pi / 2} \frac{\mathrm{d} \alpha}{\left(1-k^{2} \cos ^{2} \alpha\right)^{3 / 2}} \\
& l^{2}=(u+v)^{2}+4 h^{2}, \quad k^{2}=\frac{4 u v}{l^{2}}
\end{aligned}
$$

The kernel function is presented by means of elliptic integral. The integral equation $(45)_{2,3}$ can be solved by iterative method.

The recurrence formula is:

$$
g_{i}(u)=1+\int_{0}^{a} g_{i-1}(v) K(u, v) \mathrm{d} v, \quad g_{0}(v)=1, \quad i=1,2, \ldots n
$$

The $n$-th approximation gives with the use of kernel (49)

$$
g(u)=1+\frac{a}{a+u}\left[1-\frac{4 h}{\pi} \frac{K\left(k_{0}\right)}{l_{0}}\right]+\left(\frac{a}{a+u}\right)^{2}\left[1-\frac{4 h}{\pi} \frac{K\left(k_{0}\right)}{l_{0}}\right]^{2}+\cdots+\left(\frac{a}{a+u}\right)^{n}\left[1-\frac{4 h}{\pi} \frac{K\left(k_{0}\right)}{l_{0}}\right]^{n}
$$


where $K_{0}$ is the elliptic integral of the first kind defined by Gradshteyn and Ryzhik [17]

$$
\begin{aligned}
& K\left(k_{0}\right)=\int_{0}^{\pi / 2} \frac{\mathrm{d} \alpha}{\left(1-k_{0}^{2} \cos ^{2} \alpha\right)^{1 / 2}} \\
& l_{0}^{2}=(u+a)^{2}+4 h^{2}, \quad k_{0}^{2}=\frac{4 u a}{l_{o}^{2}}
\end{aligned}
$$

The sum of infinite geometric series converges to the solution as $n \rightarrow \infty$, giving

$$
g(u)=\left[1-\frac{a}{a+u}\left(1-\frac{2}{\pi} \frac{K\left(k_{0}\right)}{l_{0} / 2 h}\right)\right]^{-1}, \quad 0 \leq u \leq a
$$

The range of convergence is given by inequality

$$
\frac{2}{\pi} K\left(k_{0}\right)<\left(2+\frac{u}{a}\right) \frac{l_{0}}{2 h}, \quad 0 \leq u \leq a
$$

and is satisfied for all of $u$ and $a / h$.

For $h \rightarrow \infty,(2 / \pi) K\left(k_{0}\right) \rightarrow 1$ and $\left(l_{0} / 2 h\right) \rightarrow 1$, while for $h \rightarrow 0$, we have the logarithmic singularity of $K\left(k_{0}\right)$ at $u=a$ :

$$
K\left(k_{0}\right) \sim \ln \frac{1}{1-\frac{2 \sqrt{a u}}{a+u}}
$$

But $h K\left(k_{0}\right) / l_{0}$ tends to zero as $a / h \rightarrow \infty$. Thus, we have the values

$$
g\left(\frac{a}{h}\right)=\frac{2}{1+\frac{2}{\pi} \frac{1}{\sqrt{1+\delta^{2}}} K\left(\frac{\delta}{\sqrt{1+\delta^{2}}}\right)}, \quad g(0)=\sqrt{1+\frac{\delta^{2}}{4}}, \quad \delta=\frac{a}{h}
$$

The values of $g(a / h)$ changes from 1 to 2 for all of $a / h$, and $g(u)$ is given explicitly by Eq. (53). The analytical solution (53) of the Fredholm integral equation (45) with the kernel function (46) is new to the author's best knowledge. It may be utilized also in the analysis of the contact and inclusion problems in context of the magneto-electro-elastic materials.

The transient kernel function $K_{d}(u, v, p)$ may be calculated as follows:

$$
K_{d}(u, v, p)=\frac{p^{2}}{c_{3}^{2}} v \int_{0}^{\infty} \frac{1-e^{-2 \gamma h}}{\gamma(s, p)+s} J_{0}(s u) J_{0}(s v) \mathrm{d} s
$$

To accelerate convergence of the improper integral in (57), we introduce the function:

$$
R(s, p)=\frac{1-e^{-2 \gamma h}}{\sqrt{s^{2}+\frac{p^{2}}{c_{3}^{2}}}+s}-\frac{s}{2\left(s^{2}+\frac{p^{2}}{c_{3}^{2}}\right)}
$$

which behaves as $O\left(1 / s^{3}\right)$ for large $s$.

Then

$$
K_{d}(u, v, p)=\frac{p^{2}}{c_{3}^{2}} v\left[\int_{0}^{\infty} R(s, p) J_{0}(s u) J_{0}(s v) \mathrm{d} s+\frac{1}{2} I_{0}\left(\frac{p}{s_{3}} u\right) K_{0}\left(\frac{p}{s_{3}} v\right)\right]
$$

since (Gradshteyn and Ryzhik [17])

$$
\int_{0}^{\infty} \frac{s}{s^{2}+\frac{p^{2}}{c_{3}^{2}}} J_{0}(s u) J_{0}(s v) \mathrm{d} s=I_{0}\left(\frac{p}{c_{3}} u\right) K_{0}\left(\frac{p}{c_{3}} v\right) ; \quad 0<u \leq v
$$


where $I_{0}(\cdot)$ and $K_{0}(\cdot)$ are the modified zero order Bessel functions of the first and second kind, respectively.

The improper integral in Eq. (59) can be evaluated by truncated integral due to rapid convergence of the integrands as $O\left(1 / s^{4}\right)$, since for large argument $s$, we have (Watson [16]):

$$
J_{0}(s u) J_{0}(s v) \approx \frac{1}{\pi s \sqrt{u v}}(\cos [s(u-v)]-\sin [s(u+v)])
$$

The integral in finite interval $0 \leq s \leq s_{0}$ is evaluated numerically by means, for example, of Simpson's rule and for the remainder, in the interval $s_{0}<s<\infty$, we integrate by part the function (61) with multiplier $s^{-3}$ and use the cosine- and sine-integrals.

The method of successive iteration gives the solution for $f(u, p)$ :

$$
f_{i}(u, p)=1-\int_{0}^{a} f_{i-1}(v, p)\left[K_{d}(u, v, p)-K(u, v)\right] \mathrm{d} v, \quad f_{0}(v, p)=1, \quad i=1,2, \ldots, n
$$

Once $f(u, p)$ in the Laplace transform domain is determined from Eq. (62), its inversion $f(u, t)$ can be determined by numerical method, which is presented in the Sect. 5.

\subsection{Dynamic field intensity factors}

The displacement, electric potential and magnetic potential on the crack plane can be expressed, in Laplace domain, in terms of unknown functions as:

$$
\begin{aligned}
w^{*}(x, h \pm, p)= & \frac{\tau_{0}+D \alpha+B \beta}{p \tilde{c}_{44}}\left[ \pm \int_{x}^{a} \frac{f(u, p) u}{\sqrt{u^{2}-x^{2}}} \mathrm{~d} u-\int_{0}^{a} f(u, p) u \mathrm{~d} u \int_{0}^{\infty} e^{-2 \gamma h} \cos (s x) J_{0}(s u) \mathrm{d} s\right] \\
\phi^{*}(x, h \pm, p)= & \pm \frac{1}{p} \int_{x}^{a}\left[\frac{\alpha\left(\tau_{0}+D \alpha+B \beta\right) f(u, p)}{\tilde{c}_{44}}+\left(e_{1} D+e_{2} B\right) g(u)\right] \frac{u}{\sqrt{u^{2}-x^{2}}} \mathrm{~d} u \\
& -\alpha \frac{\tau_{0}+D \alpha+B \beta}{p \tilde{c}_{44}} \int_{0}^{a} f(u, p) u \mathrm{~d} u \int_{0}^{\infty} e^{-2 \gamma h} \cos (s x) J_{0}(s u) \mathrm{d} s \\
& -\frac{x}{p}\left(e_{1} D+e_{2} B\right) \int_{0}^{a} g(u) \mathrm{d} \zeta \\
\psi^{*}(x, h \pm, p)= & \pm \frac{1}{p} \int_{x}^{a}\left[\frac{\beta\left(\tau_{0}+D \alpha+B \beta\right) f(u, p)}{\tilde{c}_{44}}+\left(e_{2} D+e_{3} B\right) g(u)\right] \frac{u}{\sqrt{u^{2}-x^{2}}} \mathrm{~d} u \\
& -\beta \frac{\tau_{0}+D \alpha+B \beta}{p \tilde{c}_{44}} \int_{0}^{\infty} e^{-2 \gamma h} \cos (s x) J_{0}(s u) \mathrm{d} s-\frac{x}{p}\left(e_{2} D+e_{3} B\right) \int_{0}^{a} g(u) \mathrm{d} \zeta \\
0 \leq & x a
\end{aligned}
$$

where again $D=\bar{D}-d_{0}(p)$ and $B=\bar{B}-b_{0}(p)$. Here the oblate spheroidal coordinates $\zeta$ and $\eta$ have been introduced (Rogowski $[18,19])$ :

$$
u^{2}=x^{2}\left(1+\zeta^{2}\right)\left(1-\eta^{2}\right), \quad 2 h=x \zeta \eta, \quad x \zeta \geq 0, \quad 0 \leq \eta \leq 1
$$

and the formulae (Rogowski $[18,19])$

$$
\begin{aligned}
& \int_{0}^{\infty} e^{-2 s h} \cos (s x) J_{0}(s u) \mathrm{d} s=\frac{\zeta}{x\left(\zeta^{2}+\eta^{2}\right)} \\
& u \mathrm{~d} u=x^{2} \frac{\zeta^{2}+\eta^{2}}{\zeta} \mathrm{d} \zeta
\end{aligned}
$$


The electric displacement, magnetic induction and shear stress outside of the crack surface can be expressed as follows:

$$
\begin{gathered}
\left\{\begin{array}{l}
D_{y}^{*}(x, h \pm, p) \\
B_{y}^{*}(x, h \pm, p)
\end{array}\right\}=-\left\{\begin{array}{c}
D \\
B
\end{array}\right\} \frac{1}{p} \int_{0}^{a} g(u) u \mathrm{~d} u \int_{0}^{\infty} s J_{0}(s u)\left(1-e^{-2 s h}\right) \cos (s x) \mathrm{d} s \\
\tau_{z y}^{*}(x, h \pm, p)=-\frac{\tau_{0}+D \alpha+B \beta}{p} \int_{0}^{a} f(u, p) u \mathrm{~d} u \int_{0}^{\infty} \gamma J_{0}(s u)\left(1-e^{-2 \gamma h}\right) \cos (s x) \mathrm{d} s \\
-\alpha D_{y}^{*}(x, h \pm, p)-\beta B_{y}^{*}(x, h \pm, p), \quad x>a
\end{gathered}
$$

Using the integral (Rogowski [18])

$$
\int_{0}^{\infty} e^{-2 s h} \sin (s x) J_{0}(s u) \mathrm{d} s=\frac{\eta}{x\left(\zeta^{2}+\eta^{2}\right)}
$$

Equation (66) may be rewritten as follows:

$$
\begin{aligned}
\left\{\begin{array}{l}
D_{y}^{*}(x, h \pm, p) \\
B_{y}^{*}(x, h \pm, p)
\end{array}\right\}= & -\frac{1}{p}\left\{\begin{array}{c}
D \\
B
\end{array}\right\} \frac{d}{\mathrm{~d} x} \int_{0}^{a} g(u) u \mathrm{~d} u\left[\frac{|x|}{x \sqrt{x^{2}-u^{2}}}-\frac{\eta}{x\left(\zeta^{2}+\eta^{2}\right)}\right] \\
\tau_{z y}^{*}(x, h \pm, p)= & -\frac{1}{p}\left(\tau_{0}+D \alpha+B \beta\right) \frac{d}{\mathrm{~d} x} \int_{0}^{a} f(u, p) u \mathrm{~d} u\left[\frac{|x|}{x \sqrt{x^{2}-u^{2}}}\right. \\
& \left.+\int_{0}^{\infty}\left[\frac{\gamma}{s}\left(1-e^{-2 \gamma h}\right)-1\right] J_{0}(s u) \sin (s x) \mathrm{d} s\right] \\
& -\alpha D_{y}^{*}(x, h \pm, p)-\beta B_{y}^{*}(x, h \pm, p), \quad x>a
\end{aligned}
$$

The singular terms of these quantities at the crack tips are:

$$
\begin{aligned}
\left\{\begin{array}{l}
D_{y}^{*}(x, h \pm, p) \\
B_{y}^{*}(x, h \pm, p)
\end{array}\right\} & \approx \frac{1}{p}\left\{\begin{array}{l}
D \\
B
\end{array}\right\} \frac{g(a)|x|}{\sqrt{x^{2}-a^{2}}} \\
\tau_{z y}^{*}(x, h \pm, p) & \approx \frac{1}{p}\left(\tau_{0}+D \alpha+B \beta\right) \frac{f(a, p)|x|}{\sqrt{x^{2}-a^{2}}}-\frac{1}{p}(D \alpha+B \beta) \frac{g(a)|x|}{\sqrt{x^{2}-a^{2}}}, \quad|x| \rightarrow a^{+}
\end{aligned}
$$

Since the singular field near the crack tip exhibits the inverse square-root singularity, we define the stress, electric displacement and magnetic induction dynamic intensity factors as follows:

$$
\left\{\begin{array}{l}
K_{\tau} \\
K_{D} \\
K_{B}
\end{array}\right\}=\lim _{|x| \rightarrow a^{+}} \sqrt{2(|x|-a)}\left\{\begin{array}{c}
\tau_{z y} \\
D_{y} \\
B_{y}
\end{array}\right\}
$$

The intensity factors are obtained from Eq. (69) and are:

$$
\begin{aligned}
K_{\tau} & =\sqrt{a} \frac{1}{2 \pi i} \int_{B r}\left(\tau_{0}+D \alpha+B \beta\right) \frac{f(a, p)}{p} e^{p t} \mathrm{~d} p-\alpha K_{D}-\beta K_{B} \\
K_{D} & =g(a) \sqrt{a} \frac{1}{2 \pi i} \int_{B r} \frac{D}{p} e^{p t} \mathrm{~d} p \\
K_{B} & =g(a) \sqrt{a} \frac{1}{2 \pi i} \int_{B r} \frac{B}{p} e^{p t} \mathrm{~d} p
\end{aligned}
$$


where $D=\bar{D}-d_{0}(p)$ and $B=\bar{B}-b_{0}(p)$.

Similarly, the field intensity factors associated with the crack opening displacement $w(x, h \pm, t)$, electric potential $\phi(x, h \pm, t)$ and magnetic potential $\psi(x, h \pm, t)$ across the crack near the crack front are defined and easily derived from Eq. (63):

$$
\begin{aligned}
& K_{w}(t) \triangleq \lim _{|x| \rightarrow a^{-}} \frac{w(x, h+, t)-w(x, h-, t)}{2 \sqrt{2(a-|x|)}}=\frac{\sqrt{a}}{\tilde{c}_{44}} \frac{1}{2 \pi i} \int_{B r}\left(\tau_{0}+D \alpha+B \beta\right) \frac{f(a, p)}{p} e^{p t} \mathrm{~d} p \\
& K_{\phi}(t) \triangleq \lim _{|x| \rightarrow a^{-}} \frac{\phi(x, h+, t)-\phi(x, h-, t)}{2 \sqrt{2(a-|x|)}}=\alpha K_{w}(t)+e_{1} K_{D}(t)+e_{2} K_{B}(t) \\
& K_{\psi}(t) \triangleq \lim _{|x| \rightarrow a^{-}} \frac{\psi(x, h+, t)-\psi(x, h-, t)}{2 \sqrt{2(a-|x|)}}=\beta K_{w}(t)+e_{2} K_{D}(t)+e_{3} K_{B}(t)
\end{aligned}
$$

The intensity factors are obtained as follows:

$$
\begin{aligned}
K_{D}(t) & =g(a) \sqrt{a}\left[\bar{D} H(t)-\frac{1}{2 \pi i} \int_{B r} \frac{d_{0}(p)}{p} e^{p t} \mathrm{~d} p\right] \\
K_{B}(t) & =g(a) \sqrt{a}\left[\bar{B} H(t)-\frac{1}{2 \pi i} \int_{B r} \frac{b_{0}(p)}{p} e^{p t} \mathrm{~d} p\right] \\
K_{w}(t) & =\frac{\sqrt{a}}{\tilde{c}_{44}} \frac{1}{2 \pi i} \int_{B r} \frac{\tau_{0}+\alpha\left[\bar{D}-d_{0}(p)\right]+\beta\left[\bar{B}-b_{0}(p)\right]}{p} f(a, p) e^{p t} \mathrm{~d} p \\
K_{\phi}(t) & =\alpha K_{w}+e_{1} K_{D}+e_{2} K_{B} \\
K_{\psi}(t) & =\beta K_{w}+e_{2} K_{D}+e_{3} K_{B} \\
K_{\tau}(t) & =\tilde{c}_{44} K_{w}-\alpha K_{D}-\beta K_{B}
\end{aligned}
$$

The dynamic energy release rate is derived in the following in a similar manner which is proposed by Pak [20] and McMeeking [21] in static case. The energy release rate at the crack tip is obtained from the following integral:

$$
\begin{aligned}
G= & \frac{1}{2} \lim _{\delta \rightarrow 0} \frac{1}{\delta} \int_{0}^{\delta}\left\{\tau_{y z}(r+a, 0, t) \Delta w(r+a-\delta, t)+D_{y}(r+a, 0, t) \Delta \phi(r+a-\delta, t)\right. \\
& \left.+B_{y}(r+a, 0, t) \Delta \psi(r+a-\delta, t)\right\} \mathrm{d} r
\end{aligned}
$$

where $\Delta w, \Delta \phi$ and $\Delta \psi$ are the jumps of displacement, electric potential and magnetic potential.

If $K_{w}, K_{\phi}$ and $K_{\psi}$ are the representative displacement, electric potential and magnetic potential field intensity factors defined as the limits of $\Delta w, \Delta \phi$ and $\Delta \psi$ when $\delta \rightarrow 0$, the energy release rate is obtained as:

$$
G=\frac{1}{2}\left(K_{\tau} K_{w}+K_{D} K_{\phi}+K_{B} K_{\psi}\right)
$$

Using the solution (73) $G(t)$ is obtained as follows:

$$
G(t)=\frac{1}{2}\left(\tilde{c}_{44} K_{w}^{2}+e_{1} K_{D}^{2}+e_{3} K_{B}^{2}+2 e_{2} K_{D} K_{B}\right)
$$

Note that all field intensity factors satisfy the constitutive equations (5), which may be rewritten as:

$$
\left[K_{\tau}, K_{D}, K_{B}\right]^{\mathrm{T}}=\mathbf{C}\left[K_{w}, K_{\phi}, K_{\psi}\right]^{\mathrm{T}}
$$

This is a confirmation of the correctness of obtained results.

\subsection{Magneto-electric field of crack interior}

In this section, the solutions of electric displacement and magnetic induction inside the crack are of interest. Application of Eqs. (20) and (63) leads to: 


$$
\begin{aligned}
\frac{-\pi d_{0} \delta_{0} \sqrt{a^{2}-x^{2}}}{2 a \varepsilon_{c}}= & \int_{x}^{a}\left\{\frac{\alpha}{\tilde{c}_{44}}\left[\tau_{0}+\left(\bar{D}-d_{0}\right) \alpha+\left(\bar{B}-b_{0}\right) \beta\right] f(u, p)\right. \\
& \left.+\left[e_{1}\left(\bar{D}-d_{0}\right)+e_{2}\left(\bar{B}-b_{0}\right)\right] g(u)\right\} \frac{u}{\sqrt{u^{2}-x^{2}}} \mathrm{~d} u \\
\frac{-\pi b_{0} \delta_{0} \sqrt{a^{2}-x^{2}}}{2 a \mu_{c}}= & \int_{x}^{a}\left\{\frac{\beta}{\tilde{c}_{44}}\left[\tau_{0}+\left(\bar{D}-d_{0}\right) \alpha+\left(\bar{B}-b_{0}\right) \beta\right] f(u, p)\right. \\
& \left.+\left[e_{2}\left(\bar{D}-d_{0}\right)+e_{3}\left(\bar{B}-b_{0}\right)\right] g(u)\right\} \frac{u}{\sqrt{u^{2}-x^{2}}} \mathrm{~d} u
\end{aligned}
$$

where $d_{0}=d_{0}(p)$ and $b_{0}=b_{0}(p)$.

Differentiating both equations (77) with respect to $x$ and using the followings rule of differentiation under integral sign:

$$
\frac{\mathrm{d}}{\mathrm{d} x} \int_{x}^{a} \frac{f(u)}{\sqrt{u^{2}-x^{2}}} \mathrm{~d} u=-\frac{x f(a)}{a \sqrt{a^{2}-x^{2}}}+x \int_{x}^{a} \frac{\mathrm{d}}{\mathrm{d} u}\left(\frac{f(u)}{u}\right) \frac{\mathrm{d} u}{\sqrt{u^{2}-x^{2}}}
$$

Equation (77) may be rewritten as:

$$
\begin{aligned}
\frac{\pi d_{0} \delta_{0} x}{2 a \varepsilon_{c} \sqrt{a^{2}-x^{2}}}= & -\frac{x}{\sqrt{a^{2}-x^{2}}}\left\{\frac{\alpha}{\tilde{c}_{44}}\left[\tau_{0}+\left(\bar{D}-d_{0}\right) \alpha+\left(\bar{B}-b_{0}\right) \beta\right] f(a, p)\right. \\
& \left.+\left[e_{1}\left(\bar{D}-d_{0}\right)+e_{2}\left(\bar{B}-b_{0}\right)\right] g(a)\right\} \\
& +x \int_{x}^{a}\left\{\frac{\alpha}{\tilde{c}_{44}}\left[\tau_{0}+\left(\bar{D}-d_{0}\right) \alpha+\left(\bar{B}-b_{0}\right) \beta\right] \frac{\mathrm{d} f(u, p)}{\mathrm{d} u}\right. \\
& \left.+\left[e_{1}\left(\bar{D}-d_{0}\right)+e_{2}\left(\bar{B}-b_{0}\right)\right] \frac{\partial g(u)}{\mathrm{d} u}\right\} \frac{\mathrm{d} u}{\sqrt{u^{2}-x^{2}}} \\
\frac{\pi b_{0} \delta_{0} x}{2 a \mu_{c} \sqrt{a^{2}-x^{2}}}= & -\frac{x}{\sqrt{a^{2}-x^{2}}}\left\{\frac{\beta}{\tilde{c}_{44}}\left[\tau_{0}+\left(\bar{D}-d_{0}\right) \alpha+\left(\bar{B}-b_{0}\right) \beta\right] f(a, p)\right. \\
& \left.+\left[e_{2}\left(\bar{D}-d_{0}\right)+e_{3}\left(\bar{B}-b_{0}\right)\right] g(a)\right\} \\
& +x \int_{x}^{a}\left\{\frac{\beta}{\tilde{c}_{44}}\left[\tau_{0}+\left(\bar{D}-d_{0}\right) \alpha+\left(\bar{B}-b_{0}\right) \beta\right] \frac{\mathrm{d} f(u, p)}{\mathrm{d} u}\right. \\
& \left.+\left[e_{2}\left(\bar{D}-d_{0}\right)+e_{3}\left(\bar{B}-b_{0}\right)\right] \frac{\partial g(u)}{\mathrm{d} u}\right\} \frac{\mathrm{d} u}{\sqrt{u^{2}-x^{2}}}
\end{aligned}
$$

The terms on the left-hand sides and the first terms on the right- hand sides in both Eqs. (79) are singular at $x \rightarrow a-0$, while the second terms on the right-hand sides tends to zero in this point. For the singularity to vanish at $x \rightarrow a-0$, it must be true that:

$$
\begin{aligned}
& d_{0}=-\varepsilon_{0}\left\{\frac{\alpha}{\tilde{c}_{44}}\left[\tau_{0}+\left(\bar{D}-d_{0}\right) \alpha+\left(\bar{B}-b_{0}\right) \beta\right] f(a, p)+\left[e_{1}\left(\bar{D}-d_{0}\right)+e_{2}\left(\bar{B}-b_{0}\right)\right] g(a)\right\} \\
& b_{0}=-\mu_{0}\left\{\frac{\beta}{\tilde{c}_{44}}\left[\tau_{0}+\left(\bar{D}-d_{0}\right) \alpha+\left(\bar{B}-b_{0}\right) \beta\right] f(a, p)+\left[e_{2}\left(\bar{D}-d_{0}\right)+e_{3}\left(\bar{B}-b_{0}\right)\right] g(a)\right\}
\end{aligned}
$$

where

$$
\varepsilon_{0}=\frac{a}{\delta_{0}} \varepsilon_{c}, \quad \mu_{0}=\frac{a}{\delta_{0}} \mu_{c}
$$

Equation (80) form two coupling linear algebraic equations with respect to $d_{0}(p)$ and $b_{0}(p)$, which are dependent on the material properties, applied loadings and the dielectric permittivity and magnetic permeability of crack interior. In addition, it is found that since $f(a, p)$ and $g(a)$ depends on the ratio $a / h$, the length of a crack and the location of it have effects on $d_{0}$ and $b_{0}$, which of course depend on time. 
Thus,

$$
\begin{aligned}
\left(\bar{D}-d_{0}\right)\left[1-\varepsilon_{0} \frac{\alpha^{2}}{\tilde{c}_{44}} f(a, p)-\varepsilon_{0} e_{1} g(a)\right]-\left(\bar{B}-b_{0}\right)\left[\varepsilon_{0} \frac{\alpha \beta}{\tilde{c}_{44}} f(a, p)+\varepsilon_{0} e_{2} g(a)\right] \\
=\bar{D}+\varepsilon_{0} \frac{\alpha}{\tilde{c}_{44}} \tau_{0} f(a, p) \\
\quad-\left(\bar{D}-d_{0}\right)\left[\mu_{0} e_{2} g(a)+\mu_{0} \frac{\alpha \beta}{\tilde{c}_{44}} f(a, p)\right]+\left(\bar{B}-b_{0}\right)\left[1-\mu_{0} \frac{\beta^{2}}{\tilde{c}_{44}} f(a, p)-\mu_{0} e_{3} g(a)\right] \\
=\bar{B}+\mu_{0} \frac{\beta}{\tilde{c}_{44}} \tau_{0} f(a, p)
\end{aligned}
$$

The solution is:

$$
\begin{aligned}
\bar{D}-d_{0}= & {\left[\bar{D}\left(1-\frac{\mu_{0} \beta^{2}}{\tilde{c}_{44}} f-\mu_{0} e_{3} g\right)+\bar{B} \varepsilon_{0}\left(\frac{\alpha \beta}{\tilde{c}_{44}} f+e_{2} g\right)+\frac{\tau_{0} \varepsilon_{0} f}{\tilde{c}_{44}}\left[\alpha+\mu_{0} e_{15}\left(e_{1} e_{3}-e_{2}^{2}\right) g\right]\right] } \\
& \times\left[1-\frac{\varepsilon_{0} \alpha^{2}+\mu_{0} \beta^{2}}{\tilde{c}_{44}} f-\left(\varepsilon_{0} e_{1}+\mu_{0} e_{3}\right) g+\varepsilon_{0} \mu_{0}\left(e_{1} e_{3}-e_{2}^{2}\right) g\left[g-f\left(1-\frac{c_{44}}{\tilde{c}_{44}}\right)\right]\right]^{-1} \\
\bar{B}-b_{0}= & {\left[\bar{B}\left(1-\frac{\varepsilon_{0} \alpha^{2}}{\tilde{c}_{44}} f-\varepsilon_{0} e_{1} g\right)+\bar{D} \mu_{0}\left(\frac{\alpha \beta}{\tilde{c}_{44}} f+e_{2} g\right)+\frac{\tau_{0} \mu_{0} f}{\tilde{c}_{44}}\left[\beta+\varepsilon_{0} e_{15}\left(e_{1} e_{3}-e_{2}^{2}\right) g\right]\right] } \\
& \times\left[1-\frac{\varepsilon_{0} \alpha^{2}+\mu_{0} \beta^{2}}{\tilde{c}_{44}} f-\left(\varepsilon_{0} e_{1}+\mu_{0} e_{3}\right) g+\varepsilon_{0} \mu_{0}\left(e_{1} e_{3}-e_{2}^{2}\right) g\left[g-f\left(1-\frac{c_{44}}{\tilde{c}_{44}}\right)\right]\right]^{-1}
\end{aligned}
$$

where $f \equiv f(a, p)$ and $g \equiv g(a)$

The field intensity factors are obtained by substitution of (83) into (73), and energy release rate are obtained from Eq. (75).

Substituting the result (80), (81) into assumption (18), we obtain in Laplace transform domain

$$
\begin{aligned}
\left\{\begin{array}{l}
E_{y}^{c} \\
H_{y}^{c}
\end{array}\right\}^{*}= & -\frac{a}{\delta_{0}} \\
& \times\left\{\left\{\begin{array}{l}
\alpha \\
\beta
\end{array}\right\} \frac{1}{\tilde{c}_{44}}\left[\tau_{0}+\left(\bar{D}-d_{0}\right) \alpha+\left(\bar{B}-b_{0}\right) \beta\right] f(a, p)\right. \\
& \left.+\left[\left\{\begin{array}{l}
e_{1} \\
e_{2}
\end{array}\right\}\left(\bar{D}-d_{0}\right)+\left\{\begin{array}{l}
e_{2} \\
e_{3}
\end{array}\right\}\left(\bar{B}-b_{0}\right)\right] g(a)\right\}
\end{aligned}
$$

\subsection{Solutions based on ideal crack-face boundary conditions}

Four ideal crack-face electro-magnetic boundary conditions are the limiting cases of the electromagnetically dielectric crack model:

(i) fully impermeable case: $\varepsilon_{0} \rightarrow 0$ and $\mu_{0} \rightarrow 0$

$\bar{D}-d_{0} \rightarrow \bar{D}, \quad \bar{B}-b_{0} \rightarrow \bar{B}$ and the intensity factors are given by:

$$
\begin{aligned}
& K_{w}^{\text {imp.imp }}(t)=\frac{\sqrt{a}}{\tilde{c}_{44}}\left(\tau_{0}+\bar{D} \alpha+\bar{B} \beta\right) \frac{1}{2 \pi i} \int_{B r} \frac{f(a, p)}{p} e^{p t} \mathrm{~d} p \\
& K_{\tau}^{\text {imp.imp }}(t)=\tilde{c}_{44} K_{w}^{\text {imp.imp }}-\alpha K_{D}^{\text {imp.imp }}-\beta K_{B}^{\text {imp.imp }} \\
& K_{D}^{\text {imp.imp }}=\bar{D} g(a) \sqrt{a} H(t) \\
& K_{B}^{\text {imp.imp }}=\bar{B} g(a) \sqrt{a} H(t) \\
& K_{\phi}^{\text {imp.imp }}(t)=\alpha K_{w}^{\text {imp.imp }}(t)+e_{1} K_{D}^{\text {imp.imp }}(t)+e_{2} K_{B}^{\text {imp.imp }}(t) \\
& K_{\psi}^{\text {imp.imp }}(t)=\beta K_{w}^{\text {imp.imp }}(t)+e_{2} K_{D}^{\text {imp.imp }}(t)+e_{3} K_{B}^{\text {imp.imp }}(t)
\end{aligned}
$$

Equation (84) together with Eq. (45) indicate that $K_{\tau}, K_{w}, K_{\phi}$ and $K_{\psi}$ depend directly on the material constants, while $K_{D}$ and $K_{B}$ do not. Since $f(a, p)$ and $g(a)$ depend on the parameter of location of the crack (the 
thickness $h$ ), all field intensity factors depend on this location. Of course, these quantities depend on the level of the magneto-electro-mechanical loadings applied on the crack surface and satisfy the constitutive equations (5).

For full impermeable case, it is seen the $K_{D}$ and $K_{B}$ do not vary with time, while the $K_{w}$, in connection with other intensity factors $K_{\tau}, K_{\phi}$ and $K_{\psi}$, vary with time; in other words, the latter dynamic field intensity factors exhibit a transient character.

(ii) full permeable case: $\varepsilon_{0} \rightarrow \infty$ and $\mu_{0} \rightarrow \infty$

Then

$$
\begin{aligned}
& \left(\bar{D}-d_{0}\right) g(a)=e_{15}\left[\tau_{0}+\left(\bar{D}-d_{0}\right) \alpha+\left(\bar{B}-b_{0}\right) \beta\right] \frac{f(a, p)}{\tilde{c}_{44}} \\
& \left(\bar{B}-b_{0}\right) g(a)=q_{15}\left[\tau_{0}+\left(\bar{D}-d_{0}\right) \alpha+\left(\bar{B}-b_{0}\right) \beta\right] \frac{f(a, p)}{\tilde{c}_{44}}
\end{aligned}
$$

and

$$
\begin{aligned}
& \bar{D}-d_{0}=\frac{e_{15} \tau_{0}}{\tilde{c}_{44}} \frac{f}{g-f\left(1-\frac{c_{44}}{\tilde{c}_{44}}\right)} \\
& \bar{B}-b_{0}=\frac{q_{15} \tau_{0}}{\tilde{c}_{44}} \frac{f}{g-f\left(1-\frac{c_{44}}{\tilde{c}_{44}}\right)}
\end{aligned}
$$

where $f \equiv f(a, p)$ and $g \equiv g(a)$.

The field intensity factors are:

$$
\begin{aligned}
K_{w}^{\text {perm.perm }} & =\frac{\tau_{0}}{\tilde{c}_{44}} \sqrt{a} \frac{1}{2 \pi i} \int_{B r} \frac{1}{1-\frac{f}{g}\left(1-\frac{c_{44}}{\tilde{c}_{44}}\right)} f(a, p) e^{p t} \mathrm{~d} p \\
K_{D}^{\text {perm.perm }} & =e_{15} K_{w}^{\text {perm.perm }} \\
K_{B}^{\text {perm.perm }} & =q_{15} K_{w}^{\text {perm.perm }} \\
K_{\tau}^{\text {perm.perm }} & =c_{44} K_{w}^{\text {perm.perm }} \\
K_{\phi}^{\text {perm.perm }} & =0 \\
K_{\psi}^{\text {perm.perm }} & =0
\end{aligned}
$$

The energy release rate is

$$
G(t)=\frac{K_{\tau}^{2}}{2 c_{44}}
$$

For static case $f(a, p)=f(a)=g(a)$ and

$$
K_{w}=\frac{\tau_{0} g(a) \sqrt{a}}{c_{44}} H(t)
$$

where $g(a)$ is given by Eq. (56).

(iii) electrically impermeable and magnetically permeable: $\varepsilon_{0} \rightarrow 0$ and $\mu_{0} \rightarrow \infty \bar{D}-d_{0} \rightarrow \bar{D}, \quad d_{0} \rightarrow 0$

$$
\begin{aligned}
& K_{D}^{\text {imp.perm }}=K_{D}^{\text {imp.imp }} \\
& \bar{B}-b_{0}=\left[\bar{D}\left(e_{2} g+\frac{\alpha \beta}{\tilde{c}_{44}} f\right)+\frac{\tau_{0} \beta}{\tilde{c}_{44}}\right]\left(1+\frac{\beta^{2}}{\tilde{c}_{44}} f+e_{3} g\right)^{-1} \\
& K_{B}^{\text {imp.perm }}=g(a) \sqrt{a} \frac{1}{2 \pi i} \int_{B r} \frac{\bar{B}-b_{0}}{p} e^{p t} \mathrm{~d} p \\
& K_{w}^{\text {imp.perm }}=\frac{\tau_{0}+\bar{D} \alpha}{\tilde{c}_{44}} \sqrt{a} \frac{1}{2 \pi i} \int_{B r} \frac{f(a, p)}{p} e^{p t} \mathrm{~d} p+\beta K_{B}^{\text {imp.perm }}
\end{aligned}
$$

and $K_{\tau}, K_{\phi}$ and $K_{\psi}$ are given by Eq. (73). 
The solution for the electrically impermeable and magnetically permeable crack is independent of the applied magnetic field.

(iv) electrically permeable and magnetically impermeable: $\varepsilon_{0} \rightarrow \infty$ and $\mu_{0} \rightarrow 0 \bar{B}-b_{0} \rightarrow \bar{B}, \quad b_{0} \rightarrow 0$

$$
\begin{aligned}
& K_{B}^{\text {perm.imp }}=K_{B}^{\text {imp.imp }} \\
& \bar{D}-d_{0}=\left[\bar{B}\left(e_{2} g+\frac{\alpha \beta}{\tilde{c}_{44}} f\right)+\frac{\tau_{0} \alpha}{\tilde{c}_{44}}\right]\left(1+\frac{\alpha^{2}}{\tilde{c}_{44}} f+e_{1} g\right)^{-1} \\
& K_{D}^{\text {perm.imp }}=g(a) \sqrt{a} \frac{1}{2 \pi i} \int_{B r} \frac{\bar{D}-d_{0}}{p} e^{p t} \mathrm{~d} p \\
& K_{w}^{\text {perm.imp }}=\frac{\tau_{0}+\bar{B} \beta}{\tilde{c}_{44}} \sqrt{a} \frac{1}{2 \pi i} \int_{B r} \frac{f(a, p)}{p} e^{p t} \mathrm{~d} p+\alpha K_{D}^{\text {perm.imp }}
\end{aligned}
$$

and $K_{\tau}, K_{\phi}$ and $K_{\psi}$ are given by Eq. (73).

The solution for the electrically permeable and magnetically impermeable crack is independent of the applied electric displacement.

In practical applications, the following cases appear:

(i) let $\varepsilon_{0}$ tends to infinity and $\mu_{0}$ is finite

Then

$$
\begin{aligned}
& K_{D}^{\text {perm. } \mu_{\mathrm{c}}}=K_{D}^{\text {perm.imp }}\left[1-f_{1}(\bar{\mu}(t))\right]+K_{D}^{\text {perm.perm }} f_{1}(\bar{\mu}(t)) \\
& K_{B}^{\text {perm. } \mu_{\mathrm{c}}}=K_{B}^{\text {perm.imp }}\left[1-f_{1}(\bar{\mu}(t))\right]+K_{B}^{\text {perm.perm }} f_{1}(\bar{\mu}(t))
\end{aligned}
$$

where

$$
\begin{aligned}
& f_{1}(\bar{\mu}(t))=\frac{1}{1+\bar{\mu}(t)} \\
& \bar{\mu}(t)=\frac{\mu_{11}}{\mu_{c}} \frac{\delta_{0}}{a} \frac{1}{g(a)}\left(1+\frac{\left(q_{15}\right)^{2}}{\mu_{11} c_{44}} f(t)\right)
\end{aligned}
$$

(ii) let $\mu_{0}$ tends to infinity and $\varepsilon_{0}$ is finite

Then

$$
\begin{aligned}
K_{D}^{\varepsilon_{c} \cdot \text { perm }} & =K_{D}^{\text {imp.perm }}\left[1-f_{2}(\bar{\varepsilon}(t))\right]+K_{D}^{\text {perm.perm }} f_{2}(\bar{\varepsilon}(t)) \\
K_{B}^{\varepsilon_{c} \cdot \text { perm }} & =K_{B}^{\text {imp.perm }}\left[1-f_{2}(\bar{\varepsilon}(t))\right]+K_{B}^{\text {perm.perm }} f_{2}(\bar{\varepsilon}(t))
\end{aligned}
$$

where

$$
\begin{aligned}
& f_{2}(\bar{\varepsilon}(t))=\frac{1}{1+\bar{\varepsilon}(t)} \\
& \bar{\varepsilon}(t)=\frac{\varepsilon_{11}}{\varepsilon_{c}} \frac{\delta_{0}}{a} \frac{1}{g(a)}\left(1+\frac{\left(e_{15}\right)^{2}}{\varepsilon_{11} c_{44}} f(t)\right)
\end{aligned}
$$

In above solution, $f(t)$ is a function of time defined by:

$$
f(t)=\frac{1}{2 \pi i} \frac{1}{g(a)} \int_{B r} f(a, p) e^{p t} \mathrm{~d} p
$$

and changes from zero to one with an increase of time.

For representative magneto-electro-elastic composite $\mathrm{BaTiO}_{3}-\mathrm{CoFe}_{2} \mathrm{O}_{4}$ and first material-second material shown in Table 1 with matrices of stiffness $(107)$ and $(108)$, respectively, we have $\left(q_{15}\right)^{2} /\left(\mu_{11} c_{44}\right)=5 \times 10^{-5}$ or $5 \times 10^{-3}$ and $\left(e_{15}\right)^{2} /\left(\varepsilon_{11} c_{44}\right)=72 \times 10^{-5}$ or $115 \times 10^{-3}$, respectively, and in consequence, the relative permeability $\bar{\mu}(t)$ and permittivity $\bar{\varepsilon}(t)$ slowly increase with time.

In above equations, the notation $K^{\text {perm.imp }}$ denotes the intensity factors (73) for electrically permeable and magnetically impermeable crack boundary conditions, i.e., for the values (90). Similarly, $K^{\text {imp.perm }}$ are defined by Eqs. (73) and (89). 
Table 1 The material constants [26]

\begin{tabular}{llcrr}
\hline Properties & $\mathrm{BaTiO}_{3}$ piezo-electric & $\mathrm{CoFe}_{2} \mathrm{O}_{4}$ piezo-magnetic & First material & Second material \\
\hline$c_{44}\left(10^{9} \mathrm{~N} / \mathrm{m}^{2}\right)$ & 43.00 & 45.30 & 43.70 & 44.60 \\
$e_{15}\left(\mathrm{C} / \mathrm{m}^{2}\right)$ & 11.60 & 0.00 & 8.12 & 3.48 \\
$\varepsilon_{11}\left(10^{-9} \mathrm{C} / \mathrm{Vm}\right)$ & 11.20 & 0.08 & 7.86 & 3.42 \\
$q_{15}(\mathrm{~N} / \mathrm{Am})$ & 0.00 & 550.00 & 165.00 & 385.00 \\
$\mu_{11}\left(10^{-6} \mathrm{~N} / \mathrm{A}^{2}\right)$ & 5.00 & 590.00 & 180.50 & 414.50 \\
$d_{11}\left(10^{-9} \mathrm{C} / \mathrm{Am}\right)$ & 0.00 & 0.00 & 0.00 & 0.00 \\
\hline
\end{tabular}

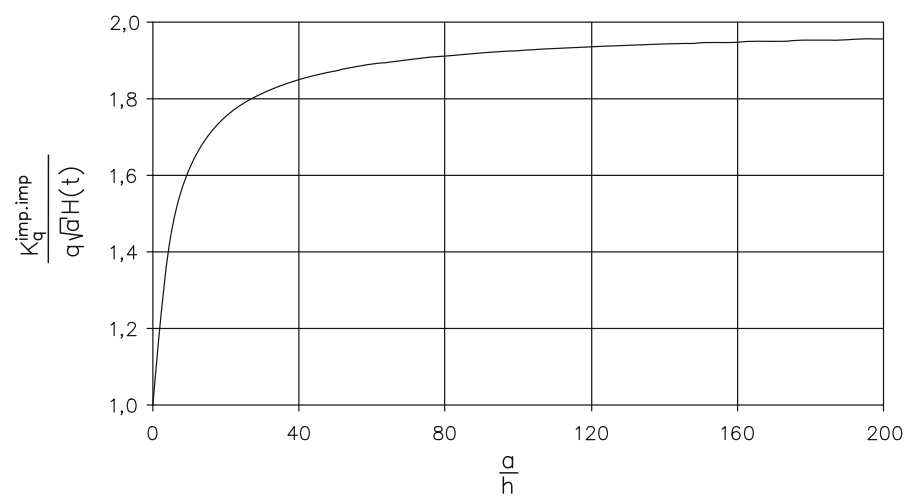

Fig. 2 The variation of the magnitude of dynamic electric displacement intensity factor and of the magnitude of dynamic magnetic induction intensity factor versus the ratio of $a / h(q$ denotes $D$ or $B)$

The functions of permittivity $\left(\varepsilon_{c}\right)$ and permeability $\left(\mu_{c}\right)$ approach zero as $\varepsilon_{c}$ and $\mu_{c}$ tend to zero and are unity as $\varepsilon_{c}$ and $\mu_{c}$ tend to infinity. The solution perfectly matches the exact solution in both limiting cases, namely permeable and/or impermeable electric and/or magnetic boundary conditions.

It is informative to consider some experimental data. Park and Sun [22] used a 0.46-mm-thick diamond wheel to cut a flaw of $11.5 \mathrm{~mm}$ length in PZT-4 piezo-electric ceramic. The notch thickness to length ratio in their test is $\delta_{0} / a \approx 0,08$. The ratio of $\varepsilon_{11} / \varepsilon_{c}$ is $\varepsilon_{11} / \varepsilon_{c}=60 / 0,0885 \approx 680$, and the function defined by Eq. (94) assumes the values: 0.0355 for $g(a)=2(h / a$ is very small) and $f(0)=0 \quad(t=0)$ and 0.0323 for $g(a)=2$ and $f(\infty)=1$.

The electric displacement intensity factor, in this case, is:

$$
K_{D}=\bar{D} g(a) \sqrt{a} H(t)\left\{\begin{array}{l}
0.9645 \\
0.9677
\end{array}\right\}+\frac{\tau_{0} e_{15}}{\tilde{c}_{44}} \frac{\sqrt{a}}{2 \pi i} \int_{B r} \frac{1}{1-\frac{f(a, p)}{g(a)}\left(1-\frac{c_{44}}{\tilde{c}_{44}}\right)} \frac{f(a, p)}{p} e^{p t} \mathrm{~d} p\left\{\begin{array}{l}
0.0355 \\
0.0323
\end{array}\right\}
$$

for very small and large time, respectively.

The quantitative result is very near to this obtained in experimental test in static case in which $f(a, p)=$ $g(a)$ and the integral part in Eq. (96) is:

$$
\frac{\tau_{0} e_{15} g(a) \sqrt{a}}{c_{44}} H(t)
$$

The quantitative result (96) shows that mechanical load produces electric displacement intensity factor. In experimental work by Park and Sun [22], the mode I crack behavior is investigated.

\section{Result and discussion}

The electric and magnetic response, in fully impermeable case, is proportional to the applied electric and magnetic load, respectively, and is independent on the mechanical loads and time, as Eq. (84), implies. The intensity factors of electric displacement and magnetic induction therefore are just a function of the geometry of the cracked MEEMs half-space as shown in Fig. 2. 


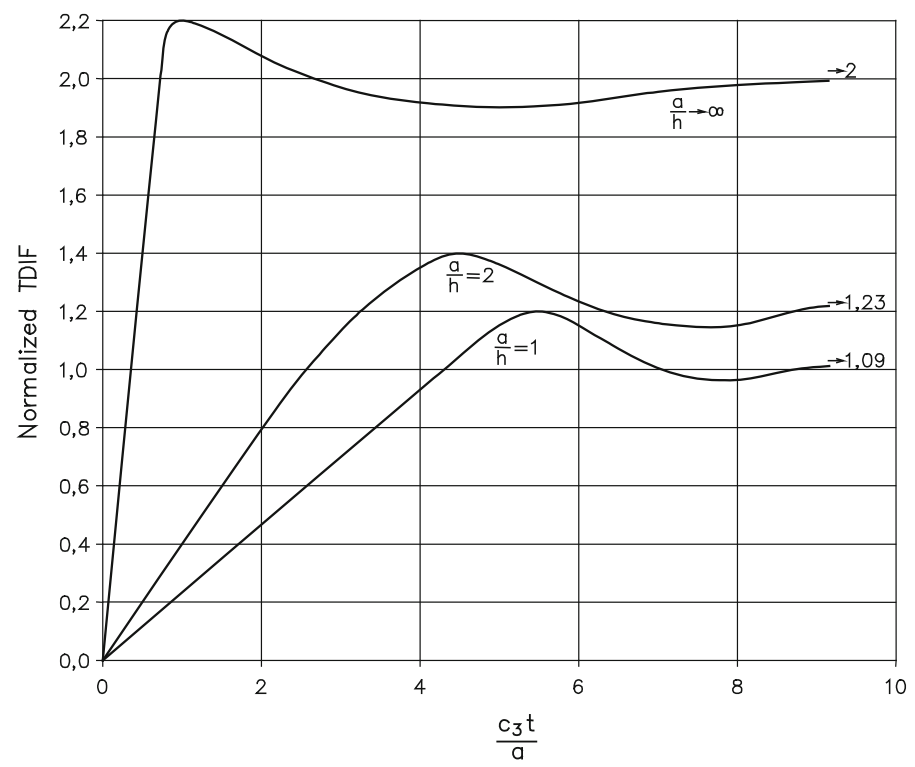

Fig. 3 Normalized tearing displacement intensity factor (TDIF) for an impermeable crack versus non-dimensional time $c_{3} t / a$; $c_{3}=\left(\tilde{c}_{44} / \rho\right)^{1 / 2}: K_{w} \tilde{c}_{44} /\left[\sqrt{a}\left(\tau_{0}+\bar{D} \alpha+\bar{B} \beta\right)\right]$

From the Fig. 2, we can see that the EDIF and MIIF increase with $a / h$. For small values of $a / h$, these quantities grow at an approximately constant rate with increasing $a / h$. For very large $a / h$, i.e., when the crack is located near the boundary of a half-space, the intensity factors of electric and magnetic fields increase slowly tending to 2 .

To obtain the dynamic intensity factors in the physical space, the inverse Laplace transform must be performed. Because of the difficulty to derive an explicit analytic solution, a numerical approach for carrying out the inversion of the Laplace transform proposed by Stehfest [23] is invoked to obtain dynamic field intensity factors in the time domain. That is, once $f(a, p)$ in the Laplace transform domain is determined numerically, its inversion $f(a, t)$ can be determined by the following scheme:

$$
f(a, t) \cong \frac{\ln (2)}{t} \sum_{n=1}^{2 L} V_{n} f\left[a, \frac{n \ln (2)}{t}\right]
$$

with

$$
V_{n}=(-1)^{n+L} \sum_{m=[(n+1) / 2]}^{\min (n, L)} \frac{m^{N}(2 m) !}{(L-m) ! m !(m-1) !(n-m) !(2 m-n) !}
$$

where $[(n+1) / 2]$ is the integer part of the real number $(n+1) / 2$ and $\mathrm{N}$ is the number of terms employed. This method not only has reasonable accuracy for a fairly wide range of Laplace transforms [24], but also is very easy and simple, as compared to other numerical inversions such as Miller and Guy [25]. In (97), only one parameter L is involved, which is suggested by Stehfest [23] to be taken as lower integers, while more than two parameters are involved to other methods for an inversion of the Laplace transform.

Figure 3 shows the response of normalized dynamic tearing displacement intensity factor (or COD intensity factor) versus non-dimensional time $c_{3} t / a$, where $c_{3}$ is defined by Eq. (10), under complex loading $\tau_{0}+\bar{D} \alpha+$ $\bar{B} \beta$, where $\bar{D}$ and $\bar{B}$ are defined by Eq. (13) and $\alpha, \beta$ by Eq. (10).

It is seen that when $a / h$ increases, the peak of TDIF increases greatly from 1,2 through 1,4 to 2,2 for $a / h=1,2, \rightarrow \infty$, respectively. In addition, the increase of $a / h(1,2, \infty)$ reduces the non-dimensional time at which TDIF reaches its peak volume from 5,5 through 4,5 to 1,0 , respectively. The values of TDIF will also arrive finally at a steady value 1,$09 ; 1,23,2$, respectively, for $a / h=1,2, \infty$, when non-dimensional time $c_{3} t / a$ is large. Note that other field intensity factors, namely $K_{\tau}, K_{\phi}$ and $K_{\psi}$, are related to $K_{w}$ as show Eq. (84). 
From the last Eq. (73), we have

$$
K_{w}=\frac{1}{\tilde{c}_{44}}\left(K_{\tau}+\alpha K_{D}+\beta K_{B}\right)
$$

Then Eq. (75) becomes

$$
\begin{aligned}
G(t)= & \frac{1}{2 \tilde{c}_{44}}\left[K_{\tau}^{2}+\left(\alpha^{2}+e_{1} \tilde{c}_{44}\right) K_{D}^{2}+\left(\beta^{2}+e_{3} \tilde{c}_{44}\right) K_{B}^{2}\right. \\
& \left.+2\left(\alpha \beta+e_{2} \tilde{c}_{44}\right) K_{D} K_{B}+2 \alpha K_{\tau} K_{D}+2 \beta K_{\tau} K_{B}\right]
\end{aligned}
$$

or in matrix form

$$
G(t)=\frac{1}{2}\left[K_{\tau}, K_{D}, K_{B}\right]^{\mathrm{T}} C^{-1}\left[K_{\tau}, K_{D}, K_{B}\right]^{\mathrm{T}}
$$

where $C^{-1}$ is a compliance matrix of MEEMs defined by Eq. (31).

Selected numerical results are presented in this section to investigate the fracture behavior of a magnetoelectro-elastic composite namely $\mathrm{BaTiO}_{3}-\mathrm{CoFe}_{2} \mathrm{O}_{4}$, and second composite made from first and second material. The volume fraction of $\mathrm{BaTiO}_{3}$ is $50 \%$ as well as first material also $50 \%$. Then the matrices of compliances are

$$
\bar{C}^{-1}=\frac{1}{2}\left(C_{1}^{-1}+C_{2}^{-1}\right)
$$

and are determined as follows

for $\mathrm{BaTiO}_{3}-\mathrm{CoFe}_{2} \mathrm{O}_{4}$ composite $(1: 1)$

$$
\bar{C}^{-1}=\left[\begin{array}{cc}
20.00 \times 10^{-12} \mathrm{~m}^{2} / \mathrm{N} 94.13 \times 10^{-4} \mathrm{~m}^{2} / \mathrm{C} 10.17 \times 10^{-6} \mathrm{Am} / \mathrm{N} \\
94.13 \times 10^{-4} \mathrm{~m}^{2} / \mathrm{C}-62,8 \times 10^{8} \mathrm{Vm} / \mathrm{C} 0 \\
10.17 \times 10^{-6} \mathrm{Am} / \mathrm{N} 0 & -1.01 \times 10^{5} \mathrm{~A}^{2} / \mathrm{N}
\end{array}\right]
$$

for first material-second material (1:1)

$$
\bar{C}^{-1}=\left[\begin{array}{l}
19.88 \times 10^{-12} \mathrm{~m}^{2} / \mathrm{N} 20.38 \times 10^{-3} \mathrm{~m}^{2} / \mathrm{C} 18.33 \times 10^{-6} \mathrm{Am} / \mathrm{N} \\
20.38 \times 10^{-3} \mathrm{~m}^{2} / \mathrm{C}-1.89 \times 10^{8} \mathrm{Vm} / \mathrm{C} 18.78 \times 10^{3} \mathrm{Am} / \mathrm{C} \\
18.33 \times 10^{-6} \mathrm{Am} / \mathrm{N} 18.78 \times 10^{3} \mathrm{Am} / \mathrm{C}-3.96 \times 10^{3} \mathrm{~A}^{2} / \mathrm{N}
\end{array}\right]
$$

Note that in both composites, piezo-electric/piezo-magnetic and first material-second material, the sums of corresponding material parameters are the same. Using the mixture rule $\kappa^{c}=\kappa V_{f}+\kappa^{\prime}\left(1-V_{f}\right)$, where $\kappa$ with superscripts $c$ without prime or prime denotes the corresponding constants in compliance matrices of composite, first material and second material and $V_{f}$ is volume fraction of first material, the matrices (103) and (104) are obtained.

The energy release rates are obtained as follows:

- composite $\mathrm{BaTiO}_{3}-\mathrm{CoFe}_{2} \mathrm{O}_{4}(1: 1)$

$$
\begin{aligned}
G= & 10.00 \times 10^{-12}\left(\mathrm{~m}^{2} / \mathrm{N}\right) K_{\tau}^{2}-3,14 \times 10^{9}(\mathrm{Vm} / \mathrm{C}) K_{D}^{2}-0.505 \times 10^{5}\left(\mathrm{~A}^{2} / \mathrm{N}\right) K_{B}^{2} \\
& +9.415 \times 10^{-3}\left(\mathrm{~m}^{2} / \mathrm{C}\right) K_{\tau} K_{D}+10,17 \times 10^{-6}(\mathrm{Am} / \mathrm{N}) K_{\tau} K_{B}
\end{aligned}
$$

- composite first material-second material $(1: 1)$

$$
\begin{aligned}
G= & 9.94 \times 10^{-12}\left(\mathrm{~m}^{2} / \mathrm{N}\right) K_{\tau}^{2}-0.945 \times 10^{8}(\mathrm{Vm} / \mathrm{C}) K_{D}^{2}-1.98 \times 10^{3}\left(\mathrm{~A}^{2} / \mathrm{N}\right) K_{B}^{2} \\
& +18.78 \times 10^{3}(\mathrm{Am} / \mathrm{C}) K_{D} K_{B}+20.38 \times 10^{-3}\left(\mathrm{~m}^{2} / \mathrm{C}\right) K_{\tau} K_{D}+18,33 \times 10^{-6}(\mathrm{Am} / \mathrm{N}) K_{\tau} K_{B}
\end{aligned}
$$

Let $x=\frac{K_{D}}{K_{\tau}} \cdot 10^{10} \mathrm{~N} / \mathrm{C}, y=\frac{K_{B}}{K_{\tau}} \cdot 10^{8} \mathrm{~A} / \mathrm{m}$. Thus, the total energy release rate is non-dimensional and is

$$
\bar{G}=\frac{G}{K_{\tau}^{2}} \cdot 10^{12} \mathrm{~N} / \mathrm{m}^{2}=\left\{\begin{array}{l}
10-31.4 x^{2}-5.05 y^{2}+0.94 x+0.1017 y \\
9.94-0.945 x^{2}-0.198 y^{2}+0.01878 x y+2.038 x+0.1833 y
\end{array}\right.
$$

for composites $\mathrm{BaTiO}_{3}-\mathrm{CoFe}_{2} \mathrm{O}_{4}$ (1:1) and first material-second material (1:1), respectively. 


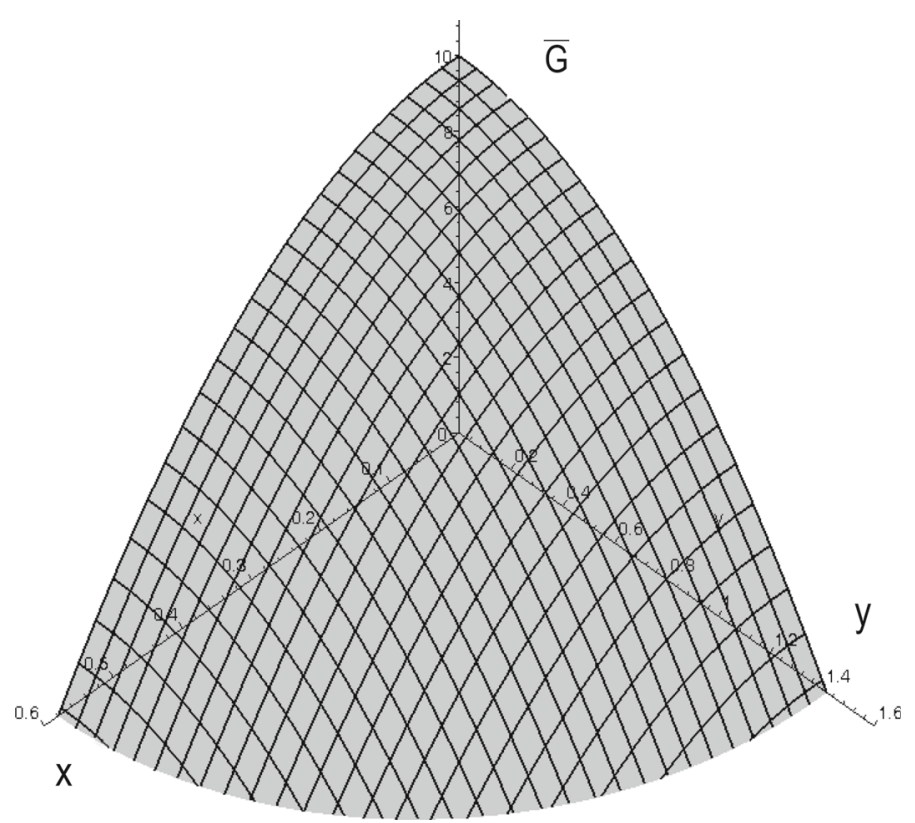

Fig. 4 Energy release rate $\bar{G}=\frac{G}{K_{\tau}^{2}} \cdot 10^{12} \mathrm{~N} / \mathrm{m}^{2}$ versus $x=\frac{K_{D}}{K_{\tau}} \cdot 10^{10} \mathrm{~N} / \mathrm{C}$ and $y=\frac{K_{B}}{K_{\tau}} \cdot 10^{8} \mathrm{~A} / \mathrm{m}$ for $\mathrm{BaTiO}_{3}-\mathrm{CoFe}_{2} \mathrm{O}_{4}$ composite

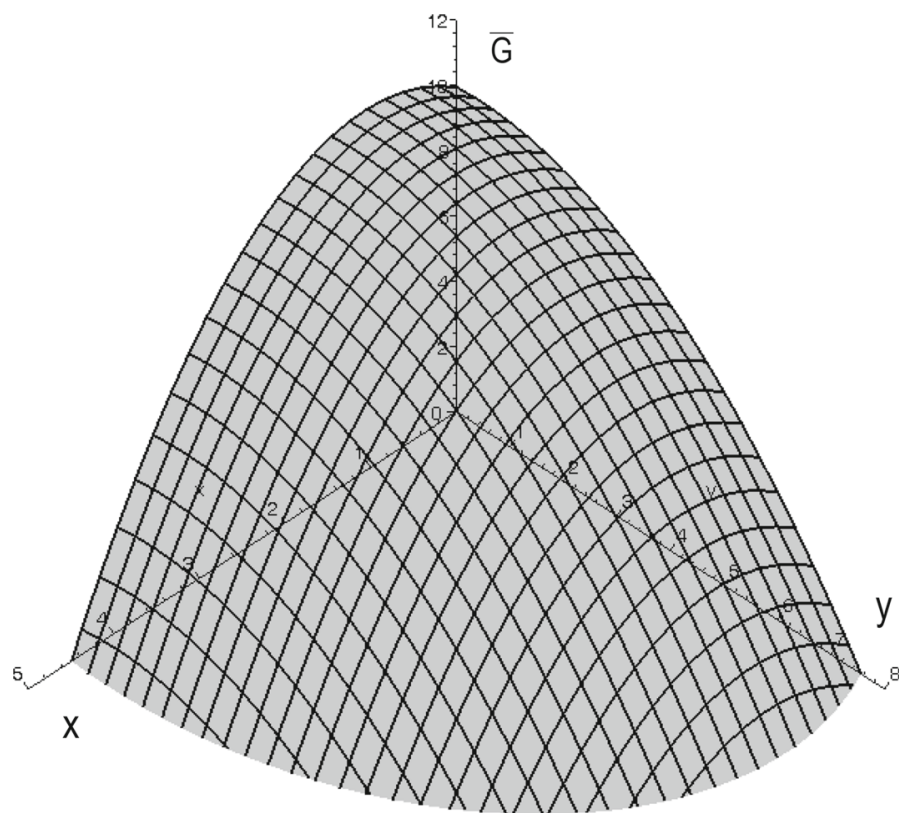

Fig. 5 Energy release rate $\bar{G}=\frac{G}{K_{\tau}^{2}} \cdot 10^{12} \mathrm{~N} / \mathrm{m}^{2}$ versus $x=\frac{K_{D}}{K_{\tau}} \cdot 10^{10} \mathrm{~N} / \mathrm{C}$ and $y=\frac{K_{B}}{K_{\tau}} \cdot 10^{8} \mathrm{~A} / \mathrm{m}$ for first material-second material composite

Figure 4 shows the effect of applied electric and magnetic fields on the energy release rate $\bar{G}$ (non-dimensional values) for composite $\mathrm{BaTiO}_{3}-\mathrm{CoFe}_{2} \mathrm{O}_{4}$.

Figure 5 shows this effect for composite made by first material-second material. It is seen that the applied electric and/or magnetic loading $D_{0}$ and $B_{0}$ (or $E_{0}$ and $H_{0}$, see Eq. 13) retard the growth of the mode III crack. The energy release rate $\mathrm{G}$ is affected by electro-magneto-elastic properties of the two constituents of composites. Note that, the matrices (103) and (104) of generalized compliances of both composites are dissimilar. Also, the matrices of generalized stiffness will be dissimilar since they are the inverse matrices of matrices (103) and (104), respectively. These are: for $\mathrm{BaTiO}_{3}-\mathrm{CoFe}_{2} \mathrm{O}_{4}$ composite (1:1) 


$$
\bar{C}=\left[\begin{array}{lll}
49.96 \times 10^{9} \mathrm{~N} / \mathrm{m}^{2} & 74.82 \times 10^{-3} \mathrm{C} / \mathrm{m}^{2} & 5.04 \mathrm{~N} / \mathrm{Am} \\
74.82 \times 10^{-3} \mathrm{C} / \mathrm{m}^{2} & -0.159 \times 10^{-9} \mathrm{C} / \mathrm{Vm} & 7.55 \times 10^{-12} \mathrm{C} / \mathrm{Am} \\
5.04 \mathrm{~N} / \mathrm{Am} & 7.55 \times 10^{-12} \mathrm{C} / \mathrm{Am} & -9.91 \times 10^{-6} \mathrm{~N} / \mathrm{A}^{2}
\end{array}\right]
$$

for first material-second material (1:1)

$$
\bar{C}=\left[\begin{array}{lll}
45.08 \times 10^{9} \mathrm{~N} / \mathrm{m}^{2} & 4.89 \mathrm{C} / \mathrm{m}^{2} & 231.8 \mathrm{~N} / \mathrm{Am} \\
4.89 \mathrm{C} / \mathrm{m}^{2} & -4.77 \times 10^{-9} \mathrm{C} / \mathrm{Vm} & 1.36 \times 10^{-12} \mathrm{C} / \mathrm{Am} \\
231.8 \mathrm{~N} / \mathrm{Am} & 1.36 \times 10^{-12} \mathrm{C} / \mathrm{Am} & -251 \times 10^{-6} \mathrm{~N} / \mathrm{A}^{2}
\end{array}\right]
$$

Due to the absence of magnetoelectric coupling coefficient in a single-phase piezo-electric and piezomagnetic material, the magneto-electric constant $d_{11}$ existing only in the piezo-electric / piezo-magnetic composite as a significant new feature cannot be determined by the mixture rule for stiffnesses. Therefore, based on the analysis of micromechanics, this coefficient is obtained as $d_{11}=1,36 \times 10^{-12} \mathrm{C} / \mathrm{Am}$ for firstsecond combination of materials and 7, $55 \times 10^{-12} \mathrm{C} / \mathrm{Am}$ for barium titanate-cobalt iron oxide bi - material. This is magnetoelectric coupling effect in composite of piezo-electric and piezo-magnetic phases.

From the Eqs. (105) and (106), it is shown that $G(t)$ is not always negative even under pure electric and/or magnetic loadings since $K_{\tau}$ relates to electric and/or magnetic loading as shown in equation (71) 1 . This main draw back in static magneto-electro-elasticity no longer exists in the dynamic case. The critical value of $G(t)$ can be measured experimentally for MEEMs, and they have been done for metals.

\section{Conclusions}

- Interesting observation from Figs. 4 and 5 is that the energy release rate $\mathrm{G}$ is affected by electro-magnetoelastic properties of the two constituents of the composites. The applied electric and/or magnetic loading $D_{0}$ and $B_{0}$ (or $E_{0}$ and $H_{0}$ ) retard the growth (the propagation) of the mode III crack.

- The solution for field intensity factors for the electrically impermeable and magnetically permeable crack is independent of the applied magnetic field.

- The solution for field intensity factors for the electrically permeable and magnetically impermeable crack is independent of the applied electric displacement.

- For full impermeable case, the $K_{D}$ and $K_{B}$ do not vary with time, while the $K_{w}$, in connection with other intensity factors $K_{\tau}, K_{\phi}$ and $K_{\psi}$, vary with time.

- Note that the plane $x=0$ is a plane of symmetry $\left(\tau_{x z}=0, \quad D_{x}=0, \quad B_{x}=0\right.$ on this plane). In consequence, the solutions are valid for quarter-plane with edge crack of length $a$.

Open Access This article is distributed under the terms of the Creative Commons Attribution License which permits any use, distribution, and reproduction in any medium, provided the original author(s) and the source are credited.

\section{References}

1. Ueda, S.: Transient dynamic response of a coated piezoelectric strip with a vertical crack. Eur. J. Mech. A Solid 22(6), 925942 (2003)

2. Nishioka, T., Shen, S.P., Yu, J.H.: Dynamic J integral, separated dynamic J integral and component separation method for dynamic interfacial cracks in piezoelectric biomaterials. Int. J. Fract. 122(3-4), 101-130 (2003)

3. Jin, B., Soh, A.K., Zhong, Z.: Propagation of an anti-plane moving crack in a functionally graded piezoelectric strip. Arch. Appl. Mech. 73(3-4), 252-260 (2003)

4. Li, X.F., Tang, G.J.: Transient response of a piezoelectric ceramic strip with an eccentric crack under electromechanical impacts. Int. J. Solids Struct. 40(13-14), 3571-3588 (2003)

5. He, T.H.: Steady propagate crack in a transverse isotropic piezoelectric material considering the permittivity of the medium in the crack gap. Int. J. Fract. 118(3), 239-249 (2002)

6. Ricci, V., Shukla, A., Chalivendra, V.B., Lee, K.H.: Subsonic interfacial fracture using strain gages in isotropic-orthotropic biomaterial. Theor. Appl. Fract. Mech. 39(2), 143-161 (2003)

7. Chen, Z.T., Worswick, M.J.: Dynamic fracture behavior of a cracked piezoelectric half space under anti-plane mechanical and in-plane electric impact. Arch. Appl. Mech. 72, 1-12 (2002)

8. Wang, B.L., Mai, Y.W.: Closed form solution for an antiplane interface crack between two dissimilar magnetoelectroelastic layers. J. Appl. Mech. Trans. ASME 73, 281-290 (2006) 
9. Li X.F.: Dynamic analysis of a cracked magnetoelectroelastic medium under antiplane mechanical and inplane electric and magnetic impacts. Int. J. Solids Struct. 42, 3185-3205 (2005)

10. Tian, W.Y., Rajapakse, R.K.N.D.: Fracture analysis of magnetoelectroelastic solids by using path independent integrals. Int. J. Fract. 131, 311-335 (2005)

11. Tian, W.Y., Rajapakse, R.K.N.D.: Fracture parameters of a penny-shaped crack at the interface of a piezoelectric bi-material system. Int. J. Fract. 141, 37-48 (2006)

12. Stroh, A.N.: Dislocations and cracks in anisotropic elasticity. Philos. Mag. 3, 625-646 (1958)

13. Parton, V.Z., Kudryavtsev, B.A.: Electromagnetoelasticity. Gordon and Breach Science Publishers, New York (1988)

14. Ma, C.C., Chen, S.K.: Exact transient analysis of an anti-plane semi-infinite crack subjected to dynamic body forces. Wave Motion 17, 161-171 (1993)

15. Rogowski, B.: The limited electrical permeable crack model in linear piezoelectricity. Int. J. Press. Vessels Pip. 84, 572-581 (2007)

16. Watson, G.N.: Theory of Bessel Functions. Cambridge University Press, Cambridge (1962)

17. Gradshteyn, I.S., Ryzhik, L.M.: Tables of Integrals, Series and Products. Academic Press, New York (1965)

18. Rogowski, B.: Fracture Mechanics of Anisotropic Bodies. Methods of Analysis and Solutions of Crack Problems. Lodz University of Technology Press, Monographs, Lodz (2014)

19. Rogowski, B.: Crack Problems in Anisotropic Thermoelasticity, Lodz University of Technology Press, Monographs, Lodz (2014)

20. Pak, Y.E.: Crack extension force in a piezoelectric material. J. Appl. Mech. Trans. ASME 57, 647-653 (1990)

21. McMeeking, R.M.: Crack tip energy release rate for a piezoelectric compact tension specimen. Eng. Fract. Mech. 64, 217-244 (1999)

22. Park, S.B., Sun, C.T.: Fracture criteria for piezoelectric ceramics. J. Am. Ceram. Soc. 78, 1475-1480 (1995)

23. Stehfest, H.: Numerical inversion of Laplace transforms. Commun. ACM 13, 47-49 (1970)

24. Davies, B., Martin, B.: Numerical inversion of the Laplace transform: a survey and comparison of methods. J. Comput. Phys. 33, 1-32 (1979)

25. Miller, M.K., Guy, W.T.: Numerical inversion of the Laplace transform by use of Jacobi polynomials. SIAM J. Numer. Anal. 3, 624-635 (1966)

26. Sih, G.C., Song, Z.F.: Magnetic and electric poling effects associated with crack growth in $\mathrm{BaTiO}_{3}-\mathrm{CoFe}_{2} \mathrm{O}_{4}$ composite. J. Theor. Appl. Fract. Mech. 39, 209-227 (2003) 